\title{
The Toxicology Investigators Consortium Case Registry—the 2019 Annual Report
}

\author{
Meghan B. Spyres ${ }^{1,2} \cdot$ Lynn A. Farrugia ${ }^{3} \cdot$ A. Min Kang ${ }^{2,4} \cdot$ Kim Aldy $^{5,6} \cdot$ Diane P. Calello ${ }^{7}$. Sharan L. Campleman ${ }^{6}$. \\ Shao $\mathrm{Li}^{6} \cdot$ Gillian A Beauchamp ${ }^{8}$. Timothy Wiegand ${ }^{9} \cdot$ Paul M. Wax ${ }^{5,6} \cdot$ Jeffery Brent ${ }^{10} \cdot$ On behalf of the Toxicology \\ Investigators Consortium Study Group
}

Received: 6 August 2020 / Revised: 21 August 2020 / Accepted: 21 August 2020

(C) American College of Medical Toxicology 2020, corrected publication 2021

\begin{abstract}
The Toxicology Investigators Consortium (ToxIC) Registry was established by the American College of Medical Toxicology (ACMT) in 2010. The Registry collects data from participating sites with the agreement that all bedside medical toxicology consultation will be entered. This tenth annual report summarizes the Registry's 2019 data and activity with its additional 7177 cases. Cases were identified for inclusion in this report by a query of the ToxIC database for any case entered from 1 January to 31 December 2019. Detailed data was collected from these cases and aggregated to provide information which included demographics, reason for medical toxicology evaluation, agent and agent class, clinical signs and symptoms, treatments and antidotes administered, mortality, and whether life support was withdrawn. $50.7 \%$ of cases were female, $48.5 \%$ were male, and $0.8 \%$ were transgender. Non-opioid analgesics was the most commonly reported agent class, followed by opioid and antidepressant classes. Acetaminophen was once again the most common agent reported. There were 91 fatalities, comprising $1.3 \%$ of all Registry cases. Major trends in demographics and exposure characteristics remained similar to past years' reports. Sub-analyses were conducted to describe exposures in cases of self-harm, gender differences in substance use disorder, and trends in addiction medicine and pain management consultations.
\end{abstract}

Keywords Poisoning $\cdot$ Overdose $\cdot$ Surveillance $\cdot$ Epidemiology $\cdot$ Medical toxicology

\section{Introduction}

The year 2019 marked the 10th full year of operation of the Toxicology Investigators Consortium (ToxIC), symbolized by continued growth, robust data collection, and an expanding research program. In 2019, the Registry also rapidly deployed a focused clinical surveillance tool centered on the evolution of vaping-related pulmonary injury and continued to generate peer-reviewed publications and presentations at national and international meetings. The

Supervising Editor: Mark B. Mycyk, MD

Electronic supplementary material The online version of this article (https://doi.org/10.1007/s13181-020-00810-7) contains supplementary material, which is available to authorized users.

Meghan B. Spyres

mspyres@gmail.com

Extended author information available on the last page of the article current report provides data on our clinical experience over 2019.

Beyond a compilation of our clinical data, this year we are highlighting some of our experiences in the important realm of self-harm attempts, substance abuse, and addiction medicine. Specifically, we are examining (1) differences in substances used for self-harm attempts based on age; (2) the gender-related experience in patients we have cared for with substance-misuse-related diagnoses; and (3) year-to-year trends and reasons for addiction medicine consultations.

In 2019, 7177 individual cases were entered into the ToxIC database deriving from 37 sites comprised of 70 separate health care facilities. By December 31, 2019, there were a total of 73,340 cases in the ToxIC database.

Nine full ToxIC publications spanning four journals were published in 2019, and 12 published ToxIC abstracts were presented at both national and international meetings. These are enumerated on the ToxIC website: https: www. ToxICRegistry.org. 
The following new ToxIC research projects were approved and initiated in 2019:

1. Comparison of physostigmine and dexmedetomidine for treatment of delirium in suspected anticholinergic poisoning

2. Treatment interventions in calcium channel blocker toxicity

3. Poisonings among transgender patients

4. Characterization of Z-drug toxicity

5. Assessment of whether the addition of dantrolene sodium to benzodiazepines in treating neuroleptic malignant syndrome improves clinical outcome

6. Methamphetamine, cocaine, and other drugs of abuse co-exposures

7. An assessment of the utility of provoked urine specimens

8. Toxic hyperthermia and the risk factors associated with increased morbidity and mortality

9. High-dose insulin euglycemia therapy use in pediatric patients with calcium channel blocker overdose

10. Clozapine-induced myocarditis and troponin elevation

11. Methylene blue usage in medical toxicology practice

12. Characteristics of kratom poisoning in the USA

13. Pulmonary puzzle: depicting patterns of use and presenting symptoms in vaping associated pulmonary injury

2019 saw the dramatic onset of a novel respiratory illness related to vaping. In response, ToxIC designed a focused intensive data collection on this topic with input from stakeholders including the US Food and Drug Administration (FDA). That study will be published separately; however, a short overview of the data collected is presented here.

The ToxIC consortium continued to expand its collaboration with the FDA on areas of interest to the Agency; 2019 was the third year of this collaboration.

In addition to the main Case Registry, ToxIC maintains 5 sub-registries representing detailed and highly focused data collections. The topics of these sub-registries are:

1. North American snakebites

2. Pediatric marijuana and opioid toxicity

3. Extracorporeal substance removal

4. Lipid resuscitation therapy

5. Plant and mushroom toxicity

2019 was the fifth and final full year of our clinical data collection for the National Institutes of Health (NIH)-funded study of the cardiovascular consequences of drug overdose. At the end of 2019, the data collection on Quality Metrics via the ToxIC Registry was terminated.

In 2019, ToxIC was supported by the NIH, the FDA, and a corporate contract with BTG International.

\section{Methods}

The operation, Health Insurance Portability and Accountability Act compliance, and institutional review board approvals have been described in prior publications. ${ }^{1,2}$

A new query was added to the Case Registry to capture likely cases of vaping-related pulmonary disease. If answered in the affirmative, the investigator is directed to a supplementary data entry form with fields related to the type of substance vaped, time frames of use and symptom onset, frequency of use, flavorings of the substance(s) vaped, source of cartridges, other substances used, relevant medical history, symptoms, details of diagnostic testing, treatments, and outcomes.

Because the data collection on quality metrics in medical toxicology was terminated at the end of 2019, associated questions on the data base were removed.

\section{Results}

In 2019, there were a total of 7177 cases reporting toxicologic exposures to the ToxIC Registry from 37 sites. This is an increase in total cases compared to $2018 .^{3}$ Table 1 lists all individual sites that contributed cases in 2019.

\section{Demographics}

Tables 2 and 3 summarize selective demographics for age and gender, and race and ethnicity, respectively. In 2019, $50.7 \%$ of cases involved female patients and $0.8 \%$ involved transgender or gender-non-conforming patients (38 female to male, 16 male to female, 3 gender nonconforming). Sixty-eight patients $(0.9 \%)$ were pregnant. Age distribution was similar to that of previous years. ${ }^{2-4}$ The majority of patients were adults age 19-65 (62.1\%) followed by adolescents age 13-18 (20.6\%). Children ( $\leq 12$ years of age) made up $11.1 \% ; 5.8 \%$ of cases involved older adults ( $>65$ years of age).

The most commonly reported race was Caucasian (63.8\%), followed by Black/African (13.7\%) and Asian $(3.4 \%)$. Race was reported as unknown/uncertain in $15.7 \%$ of cases, a decrease from the previous 3 years. ${ }^{2-4}$ Hispanic ethnicity was reported in $12.5 \%$ of cases; $19.1 \%$ of cases reported ethnicity as unknown/uncertain. Race and ethnicity are self-reported by patients, or in cases in which a patient is unable to report, it may be determined by the examining medical toxicologist to the best of their ability.

Table 4 details the referral source of inpatient and outpatient medical toxicology encounters. The majority (53.7\%) of inpatient cases were generated by the Emergency Department, and very few cases were referred 
from poison centers $(0.7 \%)$ or outpatient physicians $(0 \%)$. Outpatient encounters were primarily referred by primary care and other outpatient physicians $(68.0 \%)$, followed by self-referrals $(10.0 \%)$. These trends were similar to those of previous years. ${ }^{2-4}$

Tables 5 and 6 describe the reason for the toxicology encounter and the details of intentional pharmaceutical exposures, respectively. Consistent with previous years, ${ }^{2-4}$ intentional pharmaceutical exposures were by far the most common reason for medical toxicology encounters $(52.4 \%)$. Addiction medicine consult was a new reason for encounter in 2018 and more than doubled in $2019(2.7 \%$ to $6.6 \%){ }^{3}$ Within the intentional pharmaceutical exposures, the majority of cases were again an attempt at self-harm $(70.5 \%)$, primarily suicide attempts $(86.2 \%)$.

Cases involving intent for self-harm $(N=2630)$ are described in detail in this year's report. Demographics in cases of self-harm differed somewhat from those of the general Registry discussed above. Notably, teens age 1318 represented a higher proportion of cases of self-harm than in that of the general Registry, making up $39.5 \%$ of the cases of self-harm. Interestingly, this age group represented only $20.6 \%$ of the general Registry. Additionally, women accounted for a larger percentage of cases of selfharm than in the general Registry $(65.0 \%$ vs $50.7 \%)$. Race demographics were similar to that of the general Registry. See Tables 7 and 8 for demographic data of cases involving self-harm.

\section{Agent Classes}

In 2019, of the 7177 cases entered into the ToxIC Registry, 2105 (29.3\%) cases involved multiple agents for a total of 9792 individual agent entries. Consistent with previous years, ${ }^{2-4}$ the non-opioid analgesic class was the most common (14.1\%) class of drugs reported. In 2019, for the first time, the opioid class became the second most common agent class reported (13.4\%), followed by the antidepressant $(10.2 \%)$ and sedative-hypnotic/muscle relaxant $(8.4 \%)$ classes. Table 9 details the contribution of each agent class to the Registry. Cholinergic and chelating agents represented new exposure classes in 2019, each with one entry.

\section{Self-Harm Intent Agent Classes}

Table 10 presents the agent classes for cases in which there was intent for self-harm. There were 2630 cases $(36.6 \%)$ reporting 4590 individual agents of exposure. More than one agent was reported in $1164(44.3 \%)$ cases. The top three agent classes were analgesic (22.8\%), antidepressant (17.1\%), and sedative hypnotic/muscle relaxant $(9.5 \%)$. Notably, opioids were the ninth most common agent class reported, representing only $3.2 \%$ of agents involving self-harm, compared to $13.4 \%$ in the general Registry.

See Table 11 for the top agent classes by age group for selfharm cases. Teens age 13-18 represented a higher proportion of cases of self-harm than in that of the general Registry. In teens, the top two agent classes were the analgesic $(29.5 \%)$ and antidepressant $(19.9 \%)$ classes, similar in ranking to all age groups for self-harm. Differing from the larger Registry, anticholinergics were reported with more frequency in cases of self-harm in teens $(12.4 \%$, the third most common class) and opioids were proportionally underrepresented $(1.2 \%)$ in this age group. Bupropion was the most common antidepressant agent involved in self-harm attempts in teens $(20.5 \%)$. There were 7 deaths in teens; the agents associated with fatalities were analgesics (acetaminophen and aspirin), anticholinergics (diphenhydramine), and herbals (caffeine).

Adults and older adults had higher relative presence of drugs of abuse (opioids, sedative hypnotics, and ethanol) compared to children and teens.

\section{Analgesics}

Table 12 presents the non-opioid analgesics, the largest class in the Registry. Acetaminophen was again the most commonly reported agent $(58.5 \%),{ }^{2-5}$ followed by ibuprofen $(12.9 \%)$ and gabapentin (10.1\%). Aspirin and acetylsalicylic acid are listed separately in the Registry; when combined, they compose the third most common agent reported $(11.6 \%)$.

\section{Opioids}

Table 13 describes the opioid class. Similar to previous years, heroin was again the most common agent in the class $(37.9 \%)^{3,4}$ The relative contribution of fentanyl increased again this year, now representing the second most common opioid reported (14.6\%). Oxycodone's relative contribution remained similar to that of last year $(12.5 \%),{ }^{3}$ but it dropped to the third most common agent reported behind fentanyl. Tramadol numbers fell this year compared to 2018 (3.9\% vs $8.1 \%){ }^{3}$ Other opioid agents remained fairly stable compared to those of prior years.

Overall, opioids represented $13.4 \%$ of agents reported to the Registry. In cases of self-harm, opioids represented $0.7 \%$ of agents for children, $1.2 \%$ for teens, $4.0 \%$ for adults, and $13.3 \%$ for older adults.

\section{Antidepressants}

Table 14 describes the antidepressant class. SSRIs $(38.2 \%)$ and other antidepressants $(37.0 \%)$ represented the majority of this class. Sertraline (12.7\%) was the 
Table 1 Participating institutions providing cases to ToxIC in 2019.

\begin{tabular}{|c|c|c|}
\hline State or country & City & Hospitals \\
\hline \multirow[t]{2}{*}{ Arizona } & Phoenix & Banner-University Medical Center Phoenix \\
\hline & Phoenix & Phoenix Children’s Hospital \\
\hline \multirow[t]{10}{*}{ California } & Fresno & Community Regional Medical Center \\
\hline & Loma Linda & Loma Linda University Medical Center \\
\hline & Los Angeles & Keck Medical Center of USC \\
\hline & Los Angeles & University of Southern California Verdugo Hills \\
\hline & Sacramento & University of California Davis Medical Center \\
\hline & San Diego & Rady Children's Hospital \\
\hline & San Diego & San Diego VA Medical Center \\
\hline & San Diego & Scripps Mercy Hospital \\
\hline & San Diego & University of California San Diego-Hillcrest \\
\hline & San Diego & University of California San Diego-Thornton \\
\hline \multirow[t]{5}{*}{ Colorado } & Denver & Colorado Children's Hospital \\
\hline & Denver & Denver Health Medical Center \\
\hline & Denver & Porter and Littleton Hospital Swedish Hospital \\
\hline & Denver & Swedish Hospital \\
\hline & Denver & University of Colorado Medical Center \\
\hline Connecticut & Hartford & Hartford Hospital \\
\hline \multirow[t]{4}{*}{ Georgia } & Atlanta & Children's Healthcare of Atlanta Egleston \\
\hline & Atlanta & Children's Healthcare of Atlanta Hughes Spalding \\
\hline & Atlanta & Grady Health System \\
\hline & Atlanta & Grady Memorial Hospital \\
\hline \multirow[t]{2}{*}{ Illinois } & Evanston & Evanston North Shore University Health System \\
\hline & Chicago & UIC-Rush-Cook County Health \\
\hline \multirow[t]{4}{*}{ Indiana } & Indianapolis & IU-Eskenazi Hospital \\
\hline & Indianapolis & IU-Indiana University Hospital \\
\hline & Indianapolis & IU-Methodist Hospital-Indianapolis \\
\hline & Indianapolis & IU-Riley Hospital for Children \\
\hline Kentucky & Lexington & University of Kentucky Chandler Medical Center \\
\hline \multirow[t]{3}{*}{ Massachusetts } & Boston & Beth Israel Boston \\
\hline & Boston & Boston Children's Hospital \\
\hline & Worcester & University of Massachusetts Memorial Medical Center \\
\hline Michigan & Grand Rapids & Spectrum Health Hospitals \\
\hline Mississippi & Jackson & University of Mississippi Medical Center \\
\hline \multirow[t]{2}{*}{ Missouri } & Kansas City & Children's Mercy Hospitals and Clinics \\
\hline & St. Louis & Washington University School of Medicine in St Louis \\
\hline Nebraska & Omaha & University of Nebraska Medical Center \\
\hline New Jersey & Newark & Rutgers/New Jersey Medical School \\
\hline New Mexico & Albuquerque & University of New Mexico \\
\hline \multirow[t]{3}{*}{ New York } & Albany & Albany Medical Center \\
\hline & Rochester & Strong Memorial Hospital \\
\hline & Syracuse & Upstate Medical University-Downtown Campus \\
\hline North Carolina & Charlotte & Carolinas Medical Center \\
\hline \multirow[t]{2}{*}{ Oregon } & Portland & Doernbecher Children's Hospital \\
\hline & Portland & Oregon Health \& Science University Hospital \\
\hline \multirow[t]{4}{*}{ Pennsylvania } & Allentown & Lehigh Valley Hospital Cedar Crest \\
\hline & Allentown & Lehigh Valley Hospital Muhlenberg \\
\hline & Allentown & Lehigh Valley-17th Street \\
\hline & Philadelphia & Hahnemann University Hospital \\
\hline
\end{tabular}


Table 1 (continued)

\begin{tabular}{|c|c|c|}
\hline State or country & City & Hospitals \\
\hline & Philadelphia & Mercy Fitzgerald Hospital \\
\hline & Philadelphia & Mercy Hospital of Philadelphia \\
\hline & Philadelphia & St. Christopher's Hospital for Children \\
\hline & Pittsburgh & UPMC Children's Hospital of Pittsburgh \\
\hline & Pittsburgh & UPMC Magee Women’s Hospital \\
\hline & Pittsburgh & UPMC Mercy Hospital \\
\hline & Pittsburgh & UPMC Presbyterian/Shadyside \\
\hline \multirow[t]{6}{*}{ Texas } & Dallas & Children's Medical Center Dallas \\
\hline & Dallas & Parkland Memorial Hospital \\
\hline & Dallas & University of Texas Southwestern Clinic \\
\hline & Dallas & William P Clements University Hospital \\
\hline & Houston & Ben Taub General Hospital \\
\hline & Houston & Texas Children's Hospital \\
\hline Virginia & Richmond & Virginia Commonwealth University Medical Center \\
\hline \multirow[t]{2}{*}{ Wisconsin } & Milwaukee & Children's Hospital of Wisconsin \\
\hline & Milwaukee & Froedtert Memorial Lutheran Hospital \\
\hline \multirow[t]{3}{*}{ Canada } & Calgary & Alberta Children’s Hospital \\
\hline & & Foothills Medical Centre \\
\hline & & Peter Lougheed Centre \\
\hline Israel & Haifa & Rambam Health Care Campus \\
\hline Thailand & Bangkok & Vajira Hospital \\
\hline
\end{tabular}

most common SSRI reported, and bupropion (21.4\%) was the most common other antidepressant. In 2019, there was a decrease in other antidepressants and an increase in SNRIs reported compared to previous years. ${ }^{3,4}$

Table 2 ToxIC case demographics - age and gender.

\begin{tabular}{lc}
\hline & $N(\%)$ \\
\hline Gender & \\
Male & $3478(48.5)$ \\
Female & $3642(50.7)$ \\
Transgender & \\
Male to female & $16(0.2)$ \\
Female to male & $38(0.5)$ \\
Gender non-conforming & $3(0)$ \\
Pregnant & $68(0.9)$ \\
Age (years) & \\
$<2$ & $241(3.4)$ \\
2-6 & $327(4.6)$ \\
$7-12$ & $227(3.2)$ \\
$13-18$ & $1478(20.6)$ \\
$19-65$ & $4456(62.1)$ \\
$66-89$ & $407(5.7)$ \\
$>89$ & $11(0.2)$ \\
Unknown & $30(0.4)$ \\
Total & $7177(100)$ \\
\hline
\end{tabular}

\section{Sedative Hypnotics}

Table 15 presents the sedative hypnotic/muscle relaxant class. Benzodiazepines (primarily alprazolam $(21.1 \%)$ and clonazepam $(14.2 \%)$ ) and muscle relaxants (primarily baclofen

Table 3 ToxIC case demographics — race and Hispanic ethnicity.

\begin{tabular}{lc}
\hline & $N(\%)$ \\
\hline Race & \\
Caucasian & $4578(63.8)$ \\
Black/African & $981(13.7)$ \\
Asian & $245(3.4)$ \\
American Indian/Alaska Native & $100(1.4)$ \\
Native Hawaiian or Pacific Islander & $4(0.1)$ \\
Mixed & $130(1.8)$ \\
Other & $15(0.2)$ \\
Unknown/uncertain & $1124(15.7)$ \\
Total & $7177(100)$ \\
Hispanic ethnicity & \\
Hispanic & $900(12.5)$ \\
Non-Hispanic & $4903(68.3)$ \\
Unknown & $1374(19.1)$ \\
Total & $7177(100)$ \\
\hline
\end{tabular}

${ }^{a}$ Hispanic ethnicity as indicated exclusive of race 
Table 4 ToxIC Registry case referral sources by inpatient/ outpatient status.

\begin{tabular}{|c|c|}
\hline & $N(\%)$ \\
\hline \multicolumn{2}{|l|}{ Emergency Department (ED) or inpatient (IP) ${ }^{\mathrm{a}}$} \\
\hline ED & $3695(53.7)$ \\
\hline Admitting service & $2086(30.3)$ \\
\hline Request from another hospital service (not ED) & $451(6.6)$ \\
\hline Outside hospital transfer & $550(8.0)$ \\
\hline Poison Center & $48(0.7)$ \\
\hline Primary care provider or other outpatient treating physician & $2(0.0)$ \\
\hline Employer/independent medical evaluation & $2(0.0)$ \\
\hline Self-referral & $51(0.7)$ \\
\hline $\mathrm{ED} / \mathrm{IP}$ total & $6885(100)$ \\
\hline \multicolumn{2}{|l|}{ Outpatient $(\mathrm{OP}) /$ clinic/office consultation $^{\mathrm{b}}$} \\
\hline ED & $11(3.8)$ \\
\hline Admitting service & $0(0.0)$ \\
\hline Request from another hospital service (not ED) & $3(1.0)$ \\
\hline Outside hospital transfer & $1(0.3)$ \\
\hline Poison Center & $23(7.9)$ \\
\hline Primary care provider or other OP physician & $198(68.0)$ \\
\hline Employer/independent medical evaluation & $26(8.9)$ \\
\hline Self-referral & $29(10.0)$ \\
\hline OP total & $291(100)$ \\
\hline
\end{tabular}

(12.9\%) and cyclobenzaprine (10.8\%)) were the most common subtypes, similar to those of previous years. ${ }^{3,4}$ Other sedatives, Z-drugs, and barbiturates were again less common.

\section{Toxic Alcohols and Ethanol}

Table 16 describes data on ethanol and toxic alcohols. Ethanol was considered its own agent class, consistent with prior years and was the fifth most commonly reported agent class. After ethanol, the most commonly reported alcohols and glycols were ethylene glycol (45.3\%) and isopropanol (28.0\%). Methanol and miscellaneous alcohols each made up 13.3\% of the class.

\section{Sympathomimetics}

Table 17 presents the sympathomimetic class. Cocaine (33.8\%), methamphetamine $(33.5 \%)$, and amphetamine $(10.1 \%)$ were the most commonly reported agents in the class again this year. ${ }^{3}$

\section{Anticholinergic/Antihistamine}

Table 18 describes the anticholinergic/antihistamine class. Consistent with previous years, ${ }^{3,}{ }^{4}$ diphenhydramine
(57.1\%), followed by hydroxyzine (15.9\%), remains the most commonly reported agent in this class.

\section{Cardiovascular Agents}

Table 19 shows data on the cardiovascular class. For the third consecutive year in the Registry, sympatholytics (28.2\%) outnumber beta-blockers $(25.1 \%)$ as the most common subclass of cardiovascular agents, ${ }^{2-4}$ followed by calcium channel blockers (16.6\%). Clonidine (21.3\%) and metoprolol (9.1\%) were the most common sympatholytic and beta-blocker agents, respectively. Amlodipine (10.3\%) was the most common calcium channel blocker.

\section{Antipsychotics}

Table 20 details the antipsychotic class. Trends in the antipsychotic class were similar to those of previous years. ${ }^{3,4}$ The atypicals, led by quetiapine (46.6\%) and olanzapine (13.2\%), represent the majority of cases reported.

\section{Anticonvulsants, Mood Stabilizers, and Lithium}

Table 21 presents data on anticonvulsants, mood stabilizers, and lithium. Consistent with past years, lithium 
Table 5 Reason for medical toxicology encounter.

\begin{tabular}{ll}
\hline & $N(\%)$ \\
\hline Intentional exposure-pharmaceutical & $3550(52.4)$ \\
Intentional exposure-non-pharmaceutical & $909(12.7)$ \\
Unintentional exposure - pharmaceutical & $513(7.1)$ \\
Unintentional exposure-non-pharmaceutical & $312(4.3)$ \\
Addiction medicine consultation & $475(6.6)$ \\
Organ system dysfunction & $279(3.9)$ \\
Envenomation—-snake & $253(3.5)$ \\
Withdrawal—ethanol & $183(2.5)$ \\
Withdrawal—opioid & $152(2.1)$ \\
Interpretation of toxicology lab data & $121(1.7)$ \\
Environmental evaluation & $111(1.5)$ \\
Ethanol abuse & $92(1.3)$ \\
Occupational evaluation & $65(0.9)$ \\
Withdrawal—sedative/hypnotic & $48(0.7)$ \\
Malicious/criminal & $36(0.5)$ \\
Envenomation-spider & $35(0.5)$ \\
Withdrawal—other & $18(0.3)$ \\
Withdrawal—cocaine/amphetamine & $6(0.1)$ \\
Envenomation — scorpion & $4(0.1)$ \\
Envenomation-other & $7(0.1)$ \\
Marine /fish poisoning & $5(0.1)$ \\
Blank & $3(0.0)$ \\
Total & $7177(100)$ \\
&
\end{tabular}

Table 6 Detailed reason for encounterintentional pharmaceutical exposure.

Reason for intentional pharmaceutical exposure subgroup ${ }^{\mathrm{a}}$
Table 7 ToxIC self-

harm case

demographics - age and gender.

\begin{tabular}{ll}
\hline & $N(\%)$ \\
\hline Gender & \\
Male & $875(33.3)$ \\
Female & $1710(65.0)$ \\
Transgender & \\
Male to female & $13(0.5)$ \\
Female to male & $29(1.1)$ \\
Gender non-conforming & $3(0.1)$ \\
Pregnant & $12(0.7)$ \\
Age (years) & \\
$<2$ & $2(0.1)$ \\
$2-6$ & $6(0.2)$ \\
$7-12$ & $98(3.7)$ \\
$13-18$ & $1038(39.5)$ \\
$19-65$ & $1390(52.9)$ \\
$66-89$ & $77(2.9)$ \\
$>89$ & $3(0.1)$ \\
Unknown & $16(0.6)$ \\
Total & $2630(100)$ \\
&
\end{tabular}

was considered as its own agent class and made up just over $1 \%$ of reported agents in the Registry. Among anticonvulsants and mood stabilizers, lamotrigine and valproic acid were the most commonly reported, together making up almost half $(47.0 \%)$ of the class. Carbamazepine and phenytoin were the next most common, each making up $10.0 \%$ of agents in this class.

Table 8 ToxIC self-harm case demographics - race and Hispanic ethnicity.

$2550(70.5)$

Attempt at self-harm

$509(14.1)$

Misuse/abuse

$316(8.7)$

Therapeutic use

$241(6.7)$

Unknown

$3616(100)$

Total

Attempt at self-harm - suicidal intent subclassification $^{\mathrm{b}}$

Suicidal intent

$2189(86.2)$

Suicidal intent unknown

$270(10.6)$

No suicidal intent

$82(3.2)$

Total

$2541(100)$

${ }^{\text {a }}$ Percentage based on the total number of cases $(N=3616)$ indicating primary reason for encounter due to intentional pharmaceutical exposure

${ }^{\mathrm{b}}$ Percentage based on the total number of cases indicating attempt at self-harm $(N=$ 2541)
${ }^{\text {a }}$ Hispanic ethnicity as indicated exclusive of race

\begin{tabular}{ll}
\hline & $N(\%)$ \\
\hline Race & \\
Caucasian & $1722(65.5)$ \\
Black/African & $319(12.1)$ \\
Asian & $80(3.0)$ \\
American Indian/Alaska Native & $29(1.1)$ \\
Native Hawaiian or Pacific Islander & $1(<0.1)$ \\
Mixed & $56(2.1)$ \\
Other & $4(0.2)$ \\
Unknown/uncertain & $419(15.9)$ \\
Total & $2630(100)$ \\
Hispanic ethnicity & \\
Hispanic & $334(12.7)$ \\
Non-Hispanic & $1797(68.3)$ \\
Unknown & $499(19.0)$ \\
Total & $2630(100)$ \\
\hline
\end{tabular}


Table 9 Agent classes involved in medical toxicology consultation.

\begin{tabular}{|c|c|}
\hline & $N(\%)^{\mathrm{a}}$ \\
\hline Analgesic & $1383(14.1)$ \\
\hline Opioid & $1312(13.4)$ \\
\hline Antidepressant & $996(10.2)$ \\
\hline Sedative-hypnotic/muscle relaxant & $818(8.4)$ \\
\hline Ethanol & $705(7.2)$ \\
\hline Sympathomimetic & $633(6.5)$ \\
\hline Anticholinergic/antihistamine & $580(5.9)$ \\
\hline Cardiovascular & $525(5.4)$ \\
\hline Antipsychotic & $479(4.9)$ \\
\hline Anticonvulsant & $340(3.5)$ \\
\hline Psychoactive & $330(3.4)$ \\
\hline Envenomation and marine & $272(2.8)$ \\
\hline Diabetic medication & $147(1.5)$ \\
\hline Metals & $124(1.3)$ \\
\hline Lithium & $112(1.1)$ \\
\hline Herbal products/dietary supplements & $101(1.0)$ \\
\hline Cough and cold products & $99(1.0)$ \\
\hline Caustic & $94(1.0)$ \\
\hline Toxic alcohols & $75(0.8)$ \\
\hline Gases/irritants/vapors/dusts & $75(0.8)$ \\
\hline Plants and fungi & $67(0.7)$ \\
\hline Hydrocarbon & $64(0.7)$ \\
\hline Household products & $62(0.6)$ \\
\hline Antimicrobials & $62(0.6)$ \\
\hline Unknown agent & $55(0.6)$ \\
\hline GI & $45(0.5)$ \\
\hline Anticoagulant & $37(0.4)$ \\
\hline Chemotherapeutic and immune & $35(0.4)$ \\
\hline Insecticide & $29(0.3)$ \\
\hline Anesthetic & $28(0.3)$ \\
\hline Endocrine & $24(0.3)$ \\
\hline Other pharmaceutical product & $22(0.2)$ \\
\hline Other nonpharmaceutical product & $20(0.2)$ \\
\hline Anti-parkinsonism drugs & $9(0.0)$ \\
\hline Rodenticide & $7(0.0)$ \\
\hline Pulmonary & $7(0.0)$ \\
\hline Amphetamine-like hallucinogen & $6(0.0)$ \\
\hline Ingested foreign body & $4(0.0)$ \\
\hline Herbicide & $4(0.0)$ \\
\hline WMD/riot agent/radiological & $3(0.0)$ \\
\hline Cholinergic & $1(0.0)$ \\
\hline Chelator & $1(0.0)$ \\
\hline Total & $9792(100)$ \\
\hline
\end{tabular}

$W M D$ weapons of mass destruction

${ }^{\text {a }}$ Percentages are based on total number of reported agent entries $(N=$ 9792) in $2019 ; 2105$ cases $(29.3 \%)$ reported multiple agents
Table 10 ToxIC 2019 — ranked agent classes for cases of self-harm.

Exposure rank Totals $\%^{\mathrm{a}}$

Analgesic

$1045 \quad 22.8 \%$

Antidepressant

$785 \quad 17.1 \%$

Sedative-hypnotic/muscle relaxant $3 \quad 438 \quad 9.5 \%$

$\begin{array}{llll}\text { Anticholinergic/antihistamine } & 4 & 431 & 9.4 \%\end{array}$

$\begin{array}{llll}\text { Antipsychotic } & 5 & 348 & 7.6 \%\end{array}$

$\begin{array}{llll}\text { Cardiovascular } & 6 & 294 & 6.4 \%\end{array}$

$\begin{array}{llll}\text { Anticonvulsant } & 7 & 219 & 4.8 \%\end{array}$

$\begin{array}{llll}\text { Alcohol ethanol } & 8 & 175 & 3.8 \%\end{array}$

Opioid

Sympathomimetic $\quad 10 \quad 135 \quad 2.9 \%$

Diabetic med $\quad 11 \quad 74 \quad 1.6 \%$

Herbals/dietary supplements/vitamins $12 \quad 53 \quad 1.2 \%$

$\begin{array}{llll}\text { Cough and cold } & 13 & 51 & 1.1 \%\end{array}$

Lithium

Alcohol toxic

Caustic

Antimicrobial

GI

Household

Unknown class

$51 \quad 1.1 \%$

$40 \quad 0.9 \%$

$39 \quad 0.8 \%$

$36 \quad 0.8 \%$

$32 \quad 0.7 \%$

Anticoagulant

Metals

Endocrine

Chemotherapeutic and immune

Psychoactive

Other pharmaceuticals

Gases/vapors/irritants/dusts

Hydrocarbon

Insecticide

Anesthetic

Parkinson's med

Plants and fungi

Pulmonary

Rodenticide

Foreign objects

Herbicide

Other nonpharmaceutical

Totals

$28 \quad 0.6 \%$

$23 \quad 0.5 \%$

$20 \quad 0.4 \%$

$20 \quad 0.4 \%$

$18 \quad 0.4 \%$

$17 \quad 0.4 \%$

$17 \quad 0.4 \%$

$12 \quad 0.3 \%$

$7 \quad 0.2 \%$

$6 \quad 0.1 \%$

$6 \quad 0.1 \%$

$5 \quad 0.1 \%$

$4 \quad 0.1 \%$

$3 \quad 0.1 \%$

$3 \quad 0.1 \%$

$3 \quad 0.1 \%$

$1<0.1 \%$

$1<0.1 \%$

$1<0.1 \%$

$4590 \quad 100.0 \%$

${ }^{\mathrm{a}}$ Percentages are based on total number of reported agent entries in cases of self-harm in $2019(N=4590)$; 1164 cases $(44.3 \%)$ reported multiple agents

\section{Psychoactives}

Table 22 presents data on the psychoactive class including the amphetamine-like hallucinogen methylenedioxy 
Table 11 ToxIC 2019 — top 10 ranked agent classes for cases of self-harm by age.

\begin{tabular}{|c|c|c|c|}
\hline $\begin{array}{l}\text { Adults (19-65 years), } N=1390 \\
\text { cases }\end{array}$ & Teens ( $13-18$ years), $N=1038$ cases & Children $(0-12$ years $), N=106$ cases & $\begin{array}{l}\text { Older adults ( } \geq 66 \text { years), } N=80 \\
\text { cases }\end{array}$ \\
\hline Analgesic $(18.1 \%)^{\mathrm{a}}$ & Analgesic $(29.5 \%)^{\mathrm{b}}$ & Analgesic $(27.8 \%)^{\mathrm{c}}$ & Analgesic $(22.5 \%)^{\mathrm{d}}$ \\
\hline Antidepressant $(15.7 \%)$ & Antidepressant (19.9\%) & Antidepressant (18.5\%) & $\begin{array}{l}\text { Sedative-hypnotic/muscle relaxant } \\
\quad(14.5 \%)\end{array}$ \\
\hline $\begin{array}{l}\text { Sedative-hypnotic/muscle relaxant } \\
\quad(12.8 \%)\end{array}$ & $\begin{array}{l}\text { Anticholinergic/antihistamine } \\
\quad(12.4 \%)\end{array}$ & Anticholinergic/antihistamine (9.3)\% & Opioid $(13.3 \%)$ \\
\hline Antipsychotic $(9.3 \%)$ & Cardiovascular $(5.9 \%)$ & Cardiovascular $(7.9 \%)$ & Antidepressant (11.0\%) \\
\hline $\begin{array}{l}\text { Anticholinergic/antihistamine } \\
\quad(7.7 \%)\end{array}$ & Antipsychotic (5.5\%) & Antipsychotic $(5.3 \%)$ & Cardiovascular $(9.2 \%)$ \\
\hline Cardiovascular $(6.5 \%)$ & $\begin{array}{l}\text { Sedative-hypnotic/muscle relaxant } \\
\quad(4.4 \%)\end{array}$ & $\begin{array}{l}\text { Sedative-hypnotic/muscle relaxant } \\
(5.3 \%)\end{array}$ & $\begin{array}{l}\text { Anticholinergic/antihistamine } \\
\quad(4.6 \%)\end{array}$ \\
\hline Alcohol ethanol (5.9\%) & Anticonvulsant $(4.4 \%)$ & Anticonvulsant (4.6\%) & Anticonvulsant (4.0\%) \\
\hline Anticonvulsant $(5.1 \%)$ & Sympathomimetic (3.3\%) & Antimicrobials $(3.3 \%)$ & Antipsychotic $(4.0 \%)$ \\
\hline Opioid $(4.0 \%)$ & Cough and cold (1.7\%) & $\begin{array}{l}\text { Herbals/dietary } \\
\text { supplements/vitamins }(3.3 \%)\end{array}$ & Alcohol toxic $(3.5 \%)$ \\
\hline Sympathomimetic (2.8\%) & $\begin{array}{l}\text { Herbals/dietary } \\
\quad \text { supplements/vitamins }(1.5 \%)\end{array}$ & Cough and cold $(2.6 \%)$ & Alcohol ethanol (2.9\%) \\
\hline
\end{tabular}

a, b, c, d Percentages are based on total number of reported agent entries in each age group $2019\left(N=2570^{\mathrm{a}}, 1696^{\mathrm{b}}, 151^{\mathrm{c}}, 173^{\mathrm{d}}\right)$. Cases may report more than one agent entry. Sixteen cases of self-harm did not report age

methamphetamine (Molly). Similar to 2018, Molly exposures remained low, with 6 cases reported. ${ }^{3,4}$ Similar to the previous two years, marijuana cases (29.7\%) surpassed synthetic cannabinoid cases $(9.4 \%)$, reflecting a continued decrease in relative synthetic cannabinoid cases. ${ }^{3,4}$ This year, tetrahydrocannabinol exposures increased to $16.7 \%$ of psychoactives from $<1 \%$ in $2018 .{ }^{3}$ When combined, all non-synthetic cannabinoid product exposures represented $63.6 \%$ of the psychoactive class. Reported cases of nicotine $(7.9 \%)$ increased again in 2019 , up from $3.1 \%$ the previous year. ${ }^{3}$

Table 12 Analgesics.

\begin{tabular}{ll}
\hline & $N(\%)$ \\
\hline Acetaminophen & $809(58.5)$ \\
Ibuprofen & $178(12.9)$ \\
Gabapentin & $140(10.1)$ \\
Aspirin & $95(6.9)$ \\
Acetylsalicylic acid & $66(4.8)$ \\
Naproxen & $27(2.0)$ \\
Pregabalin & $16(1.2)$ \\
Salicylic acid & $16(1.2)$ \\
Analgesic unspecified & $12(0.9)$ \\
Meloxicam & $6(0.4)$ \\
Miscellaneous & a \\
Class total & $14(1.0)$ \\
\hline
\end{tabular}

${ }^{\mathrm{a}}$ Includes aminophenazone, diclofenac, indomethacin, magnesium salicylate, mefenamic acid, methylsalicylate, oil of wintergreen, salicylamide, and unspecified NSAID

\section{Envenomations and Marine Poisonings}

Table 23 shows data on envenomations and marine poisonings. Snake envenomations represented by Crotalus (43.4\%), snake unspecified (18.8\%), and Agkistrodon (16.9\%) were the top three exposures reported to this class. Again in 2019,

Table 13 Opioids.

\begin{tabular}{ll}
\hline & $N(\%)$ \\
\hline Heroin & $497(37.9)$ \\
Fentanyl & $192(14.6)$ \\
Oxycodone & $164(12.5)$ \\
Buprenorphine & $95(7.2)$ \\
Opioid unspecified & $75(5.7)$ \\
Methadone & $74(5.6)$ \\
Hydrocodone & $55(4.2)$ \\
Tramadol & $51(3.9)$ \\
Morphine & $32(2.4)$ \\
Naloxone & $26(2.0)$ \\
Hydromorphone & $13(1.0)$ \\
Naltrexone & $8(0.6)$ \\
Codeine & $7(0.5)$ \\
Acetyl fentanyl & $5(0.4)$ \\
Miscellaneous & \\
Class total & $18(1.4)$ \\
\hline ancludes carfentanil, diphenoxylate, loperamide, meperidine, N-allyl \\
norfentanyl, norfentanyl, opioid tincture, oxymorphone, propoxyphene, \\
and tapentadol & $1312(100)$ \\
&
\end{tabular}


Loxosceles exposures were the fourth most common exposure in this class $(7.7 \%)^{3}{ }^{3,4}$

\section{Diabetic Agents}

Table 24 presents the diabetic medication agent class. Metformin was the most common agent at $32.0 \%$ of the agent class, followed by insulin (26.5\%) and glipizide (20.4\%). The sulfonylureas glipizide, glimepiride, and glyburide together made up $36.0 \%$ of the class.

\section{Metals}

Table 25 presents the metal class. Lithium is its own agent class and is reported with the anticonvulsants and mood stabilizers. Trends were similar to those of previous years with lead $(29.8 \%)$ and iron $(16.1 \%)$ representing the majority of reported cases. ${ }^{3,4}$ Notably, there was a relative decrease in

Table 14 Antidepressant agents.

\begin{tabular}{ll}
\hline & $N(\%)$ \\
\hline Selective serotonin reuptake inhibitors (SSRIs) & $381(38.2)$ \\
Sertraline & $126(12.7)$ \\
Fluoxetine & $99(9.9)$ \\
Escitalopram & $74(7.4)$ \\
Citalopram & $55(5.5)$ \\
Paroxetine & $22(2.2)$ \\
Fluvoxamine & $5(0.5)$ \\
Other antidepressants & $369(37.0)$ \\
Bupropion & $213(21.4)$ \\
Trazodone & $127(12.8)$ \\
Mirtazapine & $19(1.9)$ \\
Antidepressant unspecified & $7(0.7)$ \\
Miscellaneous & \\
Tricyclic antidepressants (TCAs) & $<5(<0.5)$ \\
Amitriptyline & $117(11.7)$ \\
Nortriptyline & $84(8.4)$ \\
Doxepin & $16(1.6)$ \\
Miscellaneous ${ }^{\mathrm{b}}$ & $13(1.3)$ \\
Serotonin-norepinephrine reuptake inhibitors (SNRIs) & $<5(<0.5)$ \\
Venlafaxine & $128(12.9)$ \\
Duloxetine & $78(7.8)$ \\
Desvenlafaxine & $41(4.1)$ \\
Miscellaneous ${ }^{\mathrm{c}}$ & $7(0.7)$ \\
Monoamine oxidase inhibitor (MAOIs) & $<5(<0.5)$ \\
Phenelzine & $1(0.1)$ \\
Class total & $1(0.1)$ \\
\hline & $996(100)$ \\
\hline
\end{tabular}

${ }^{a}$ Includes vortioxetine and tranylcypromine

${ }^{\mathrm{b}}$ Includes protriptyline, clomipramine, desipramine, and noxiptiline

${ }^{\mathrm{c}}$ Includes milnacipran and levomilnacipran
Table 15 Sedative-hypnotic/muscle relaxants by type.

\begin{tabular}{|c|c|}
\hline & $N(\%)$ \\
\hline Benzodiazepine & $443(54.3)$ \\
\hline Alprazolam & $172(21.1)$ \\
\hline Clonazepam & $116(14.2)$ \\
\hline Lorazepam & $64(7.8)$ \\
\hline Diazepam & $41(5.0)$ \\
\hline Benzodiazepine unspecified & $26(3.2)$ \\
\hline Temazepam & $15(1.8)$ \\
\hline Miscellaneous $^{\mathrm{a}}$ & $9(1.1)$ \\
\hline Muscle relaxant & $248(30.4)$ \\
\hline Baclofen & $105(12.9)$ \\
\hline Cyclobenzaprine & $88(10.8)$ \\
\hline Tizanidine & $28(3.4)$ \\
\hline Methocarbamol & $12(1.5)$ \\
\hline Carisoprodol & $12(1.5)$ \\
\hline Metaxalone & $3(0.4)$ \\
\hline Other sedatives & $59(7.2)$ \\
\hline Buspirone & $34(4.2)$ \\
\hline Sed-hypnotic/muscle relaxant unspecified & $16(2.0)$ \\
\hline Miscellaneous $^{\mathrm{b}}$ & $9(1.1)$ \\
\hline Non-benzodiazepine agonists (“Z” drugs) & $44(5.4)$ \\
\hline Zolpidem & $41(5.0)$ \\
\hline Eszopiclone & $3(0.4)$ \\
\hline Barbiturates & $22(2.7)$ \\
\hline Butalbital & $12(1.5)$ \\
\hline Phenobarbital & $5(0.6)$ \\
\hline Miscellaneous ${ }^{\mathrm{c}}$ & $5(0.6)$ \\
\hline Class total & $816(100)$ \\
\hline
\end{tabular}

${ }^{a}$ Includes chlordiazepoxide, oxazepam, triazolam, midazolam, and flurazepam

${ }^{\mathrm{b}}$ Includes phenibut (beta-phenyl-gamma-aminobutyric acid), propofol, orphenadrine, meprobamate, etizolam, and aminobutyric acid

${ }^{\mathrm{c}}$ Includes barbituate unspecified and pentobarbital

Table 16 Ethanol and toxic alcohols.

\begin{tabular}{lc}
\hline & $N(\%)$ \\
\hline Ethanol $^{\mathrm{a}}$ & $705(100)$ \\
Nonethanol alcohols and glycols & $34(45.3)$ \\
Ethylene glycol & $21(28.0)$ \\
Isopropanol & $10(13.3)$ \\
Methanol & $10(13.3)$ \\
Miscellaneous & $75(100)$ \\
Class total & \\
\hline a Ethanol is considered a separate agent class & \\
${ }^{b}$ Includes benzyl alcohol, butyl ethylene glycol, diethylene glycol, ethyl- \\
ene glycol monomethyl ether (EGME, 1-methoxyethanol), propylene \\
glycol, toxic alcohol unspecified, and triethylene glycol mono butyl ether
\end{tabular}


Table 17 Sympathomimetic agents.

\begin{tabular}{ll}
\hline & $N(\%)$ \\
\hline Cocaine & $214(33.8)$ \\
Methamphetamine & $212(33.5)$ \\
Amphetamine & $64(10.1)$ \\
Methylphenidate & $41(6.5)$ \\
Dextroamphetamine & $29(4.6)$ \\
Sympathomimetic unspecified & $17(2.7)$ \\
Lisdexamfetamine & $13(2.1)$ \\
MDMA (methylenedioxy-N-methamphetamine, Ecstasy) & $11(1.7)$ \\
Dexmethylphenidate & $5(0.8)$ \\
Pseudoephedrine & $5(0.8)$ \\
Atomoxetine & $5(0.8)$ \\
Miscellaneous & \\
Class total & $17(2.7)$ \\
\end{tabular}

${ }^{a}$ Includes phenylephrine, epinephrine, tetrahydrozoline, phentermine, methylethcathinone, norpseudoephedrine, diethylpropion, propylhexedrine, clenbuterol, and methylenedioxypyrovalerone (MDPY)

iron cases reported in 2019 compared to last year $(24.8 \%){ }^{3}$ Relative contributions of cobalt (12.1\%) and mercury (12.1\%) to the metal class increased this year (both $4.0 \%$ in 2018). ${ }^{3}$

\section{Herbal Products and Dietary Supplements}

Table 26 details herbal products and dietary supplements. Caffeine was again the most commonly reported agent

Table 18 Anticholinergi$\mathrm{c}$ and antihistamine agents.

\begin{tabular}{ll}
\hline & $N(\%)$ \\
\hline Diphenhydramine & $331(57.1)$ \\
Hydroxyzine & $92(15.9)$ \\
Doxylamine & $32(5.5)$ \\
Chlorpheniramine & $20(3.4)$ \\
Benztropine & $15(2.6)$ \\
Loratadine & $11(1.9)$ \\
Cetirizine & $11(1.9)$ \\
Promethazine & $10(1.7)$ \\
Anticholinergic unspecified & $8(1.4)$ \\
Dimenhydrinate & $8(1.4)$ \\
Dicyclomine & $7(1.2)$ \\
Pyrilamine & $6(1.0)$ \\
Miscellaneous & $29(5)$ \\
Class total & $580(100)$
\end{tabular}

${ }^{a}$ Includes brompheniramine, oxybutynin, atropine, cyproheptadine, fexofenadine, glycopyrrolate, hyoscyamine, meclizine, antihistamine unspecified, cyclopentolate, tolterodine, and solifenacin
Table 19 Cardiovascular agents.

\begin{tabular}{|c|c|}
\hline & $N(\%)$ \\
\hline Alpha-2 Agonist & $148(28.2)$ \\
\hline Clonidine & $112(21.3)$ \\
\hline Guanfacine & $35(6.7)$ \\
\hline Dexmedetomidine & $1(0.2)$ \\
\hline Beta-blockers & $132(25.1)$ \\
\hline Metoprolol & $48(9.1)$ \\
\hline Propranolol & $46(8.8)$ \\
\hline Carvedilol & $20(3.8)$ \\
\hline Atenolol & $8(1.5)$ \\
\hline Labetalol & $5(1.0)$ \\
\hline Miscellaneous $^{\mathrm{a}}$ & $5(1)$ \\
\hline Calcium channel blocker & $87(16.6)$ \\
\hline Amlodipine & $54(10.3)$ \\
\hline Diltiazem & $16(3.0)$ \\
\hline Verapamil & $11(2.1)$ \\
\hline Nifedipine & $5(1.0)$ \\
\hline Calcium channel blocker unspecified & $1(0.2)$ \\
\hline ACEI/ARB & $54(10.3)$ \\
\hline Lisinopril & $35(6.7)$ \\
\hline Losartan & $12(2.3)$ \\
\hline Miscellaneous $^{\mathrm{b}}$ & $7(1.3)$ \\
\hline Cardiac glycosides & $28(5.3)$ \\
\hline Digoxin & $28(5.3)$ \\
\hline Other antihypertensives and vasodilators & $25(4.8)$ \\
\hline Prazosin & $15(2.9)$ \\
\hline Miscellaneous $^{\mathrm{c}}$ & $10(1.9)$ \\
\hline Antidysrhythmics and other CV agents & $20(3.8)$ \\
\hline Cardiovascular agent unspecified & $6(1.1)$ \\
\hline Flecainide & $5(1.0)$ \\
\hline Miscellaneous ${ }^{\mathrm{d}}$ & $9(1.7)$ \\
\hline Diuretics & $17(3.2)$ \\
\hline Hydrochlorothiazide & $8(1.5)$ \\
\hline Furosemide & $5(1.0)$ \\
\hline Miscellaneous $^{\mathrm{e}}$ & $4(0.8)$ \\
\hline Antihyperlipidemic & $14(2.7)$ \\
\hline Atorvastatin & $7(1.3)$ \\
\hline Miscellaneous ${ }^{\mathrm{f}}$ & $7(1.3)$ \\
\hline Class total & $525(100)$ \\
\hline
\end{tabular}

${ }^{\mathrm{a}}$ Includes nadolol, timolol, nebivolol, and bisoprolol

${ }^{\mathrm{b}}$ Includes valsartan, benazepril, and enalapril

${ }^{\mathrm{c}}$ Includes isosorbide, tamsulosin, hydralazine, sacubitril, isobutyl nitrite, and doxazosin

${ }^{\mathrm{d}}$ Includes amiodarone, midodrine, propafenone, ivabradine, and dofetilide

${ }^{\mathrm{e}}$ Includes spironolactone, torsemide, and chlorthalidone

${ }^{\mathrm{f}}$ Includes simvastatin, pravastatin, rosuvastatin, lovastatin, and fenofibrate 
Table 20 Antipsychotic agents.

\begin{tabular}{ll}
\hline & $N(\%)$ \\
\hline Quetiapine & $223(46.6)$ \\
Olanzapine & $63(13.2)$ \\
Aripiprazole & $43(9.0)$ \\
Risperidone & $41(8.6)$ \\
Haloperidol & $28(5.8)$ \\
Lurasidone & $17(3.5)$ \\
Clozapine & $13(2.7)$ \\
Ziprasidone & $11(2.3)$ \\
Cariprazine & $6(1.3)$ \\
Fluphenazine & $6(1.3)$ \\
Brexpiprazole & $6(1.3)$ \\
Prochlorperazine & $5(1.0)$ \\
Paliperidone & $5(1.0)$ \\
Chlorpromazine & $5(1.0)$ \\
Miscellaneous & $7(1.5)$ \\
Class total & $479(100)$
\end{tabular}

${ }^{\text {a }}$ Includes asenapine, clotiapine, antipsychotic unspecified, thiothixene, perphenazine, and thioridazine

$(37.6 \%)^{3}$ followed by melatonin $(24.8 \%)$. Infrequently reported miscellaneous agents made up $37.6 \%$ of the agent class.

\section{Household Agents}

Table 27 describes household agents reported to the Registry. Soaps and detergents $(19.4 \%)$, cleaning solutions and

Table21 Anticonvulsants and mood stabilizers.

\begin{tabular}{ll}
\hline & $N(\%)$ \\
\hline Lithium $^{\mathrm{a}}$ & $112(100)$ \\
Lamotrigine & $88(25.9)$ \\
Valproic acid & $71(20.9)$ \\
Carbamazepine & $34(10.0)$ \\
Phenytoin & $34(10.0)$ \\
Oxcarbazepine & $30(8.8)$ \\
Topiramate & $25(7.4)$ \\
Levetiracetam & $16(4.7)$ \\
Anticonvulsant unspecified & $11(3.2)$ \\
Lacosamide & $9(2.6)$ \\
Clobazam & $6(1.8)$ \\
Divalproex & $6(1.8)$ \\
Miscellaneous & $10(2.9)$ \\
Class total & $340(100)$
\end{tabular}

${ }^{\text {a }}$ Lithium is considered a separate agent class

b Includes citalopram, fosphenytoin, perampanel, primidone, rufinamide, and zonisamide disinfectants (14.5\%), sodium hypochlorite $\leq 6 \%(14.5 \%)$, and laundry detergent pods $(14.5 \%)$ were the most commonly reported agents in this class.

\section{Gases, Irritants, Vapors, and Dusts}

Table 28 describes the gas, irritant, vapor, and dust class. Carbon monoxide was again the most commonly reported agent in this class $(49.3 \%),{ }^{2-5}$ followed by smoke $(9.3 \%)$.

\section{Plants and Fungi}

Table 29 presents data for plant and fungi exposures for the Registry in 2019. Mold was again the most common exposure (47.8\%). ${ }^{3}$ Mitragyna speciosa (kratom) (16.4\%) was the second most common exposure, up from $12.1 \%$ in $2018 .^{3}$ Infrequent miscellaneous agents made up $35.8 \%$ of the class.

\section{Cough and Cold Preparations}

Table S1 details data on cough and cold preparations reported to the Registry. Dextromethorphan was by far the most commonly reported agent, making up $74.7 \%$ of the class.

Table 22 Psychoactive agents.

\begin{tabular}{ll}
\hline & $N(\%)$ \\
\hline Molly_amphetamine-like hallucinogen ${ }^{\mathrm{a}}$ & $6(100)$ \\
Marijuana & $98(29.7)$ \\
Tetrahydrocannabinol & $55(16.7)$ \\
Cannabinoid synthetic & $31(9.4)$ \\
Cannabinoid nonsynthetic & $27(8.2)$ \\
Nicotine & $26(7.9)$ \\
Delta-9-tetrahydrocannabinol & $21(6.4)$ \\
LSD & $15(4.5)$ \\
Gamma hydroxybutyrate & $11(3.3)$ \\
Phencyclidine & $10(3.0)$ \\
Cannabidiol & $8(2.4)$ \\
Hallucinogenic amphetamines & $7(2.1)$ \\
Psychoactive unspecified & $5(1.5)$ \\
Miscellaneous & $16(4.8)$ \\
Class total & $330(100)$ \\
\hline
\end{tabular}

LSD lysergic acid diethylamide

${ }^{a}$ Amphetamine-like hallucinogens are considered a separate agent class

${ }^{\mathrm{b}}$ Includes ketamine, donepezil, methylenedioxymethamphetamine, hallucinogen unspecified, tryptamine, gamma butyrolactone, disulfram, and pharmaceutical cannabidiol 
Table 23 Envenomations.

\begin{tabular}{ll}
\hline & $N(\%)$ \\
\hline Crotalus (rattlesnake) & $118(43.4)$ \\
Snake unspecified & $51(18.8)$ \\
Agkistrodon (copperhead, cottonmouth/water moccasin) & $46(16.9)$ \\
Loxosceles (recluse spiders) & $21(7.7)$ \\
Trimeresurus unspecified (pit viper unspecified) & $7(2.6)$ \\
Latrodectus (widow spiders) & $6(2.2)$ \\
Spider unspecified & $5(1.8)$ \\
Miscellaneous & \\
Class total & $18(6.6)$ \\
\hline
\end{tabular}

${ }^{a}$ Includes Trimeresurus albolabris (var Pit viper incl white lipped, green tree), palytoxin, stingray, Centruroides (var Scorpion incl Bark), Hymenoptera (bees, wasps, ants), animal bite unspecified, ciguatera poisoning, Nerodia erythrogaster (plain-bellied water snake), scorpion unspecified, and Naja kaouthia (monocled cobra)

\section{Caustics}

Table S2 presents the caustic agent class. Sodium hypochlorite unknown concentration was the most common agent reported in this class $(13.8 \%)$.

\section{Hydrocarbons}

Table S3 presents the hydrocarbon agent class. The largest contributor to the class was unspecified hydrocarbons with $14.1 \%$ of the agent class. Petroleum distillates $(12.5 \%)$ were the next most commonly reported agents.

\section{Antimicrobials}

Table S4 presents data on antimicrobial agents. In 2019, amoxicillin was the most commonly reported agent (12.9\%) followed by valacyclovir $(9.7 \%)$ and dapsone $(8.1 \%)$.

Table 24 Diabetic medications.

\begin{tabular}{ll}
\hline & $N(\%)$ \\
\hline Metformin & $47(32.0)$ \\
Insulin & $39(26.5)$ \\
Glipizide & $30(20.4)$ \\
Glimepiride & $16(10.9)$ \\
Glyburide & $7(4.8)$ \\
Miscellaneous $^{\mathrm{a}}$ & $8(5.4)$ \\
Class Total & $147(100)$ \\
\hline
\end{tabular}

${ }^{a}$ Includes diabetic medication unspecified, diazoxide, linagliptin, liraglutide, and sulfonylurea unspecified
Table 25 Metals.

\begin{tabular}{ll}
\hline & $N(\%)$ \\
\hline Lead & $37(29.8)$ \\
Iron & $20(16.1)$ \\
Cobalt & $15(12.1)$ \\
Mercury & $15(12.1)$ \\
Arsenic & $7(5.6)$ \\
Chromium & $7(5.6)$ \\
Gadolinium & $5(4.0)$ \\
Miscellaneous & $18(14.5)$ \\
Class total & $124(100)$ \\
\hline
\end{tabular}

${ }^{a}$ Includes magnesium, thallium, metal unspecified, cadmium, barium, zinc metal, aluminum, nickel, selenium, antimony, and copper

\section{Gastrointestinal Agents}

Table S5 presents gastrointestinal agents. Ondansetron (26.7\%), omeprazole (13.3\%), and pantoprazole (11.1\%) were the most commonly reported agents. Ranitidine exposures decreased in 2019 (4.4\%) compared to last year $(20.0 \%)^{3}$

\section{Insecticides, Herbicides, Rodenticides, and Fungicides.}

Table S6 presents the pesticide (insecticide, herbicide, rodenticide, and fungicide) class. There were 28 insecticides reported, $35.7 \%$ of which were miscellaneous agents. There were 7 rodenticides reported; rodenticides unspecified composed $57.1 \%$ of rodenticides. Glyphosate represented $75 \%$ of the 4 herbicides reported. No fungicides were reported.

Table 26 Herbal agents and dietary supplements.

\begin{tabular}{|c|c|}
\hline & $N(\%)$ \\
\hline Caffeine & $38(37.6)$ \\
\hline Melatonin & $25(24.8)$ \\
\hline Miscellaneous ${ }^{\mathrm{a}}$ & 38 (37.6) \\
\hline Class total & $101(100)$ \\
\hline \multicolumn{2}{|c|}{$\begin{array}{l}\text { a Includes alpha lipoic acid, ashwangandha, citronella oil, dietary supple- } \\
\text { ment unspecified, essential oil unspecified, eucalyptus oil, eugenol (clove } \\
\text { oil), Garcinia cambogia, green tea extract, herbal (dietary) multibotanical, } \\
\text { herbals/dietary supplement/vitamins unspecified, lemongrass oil, potassi- } \\
\text { um, St. John's wort, senna, thymol, vitamin A, vitamin } B_{3} \text { (niacin), vita- } \\
\text { min } B_{9} \text { (folic acid), vitamin D, vitamin E (tocopherol), vitamin unspeci- } \\
\text { fied, and yerba mate green tea extract }\end{array}$} \\
\hline
\end{tabular}


Table 27 Household agents.

\begin{tabular}{ll}
\hline & $N(\%)$ \\
\hline Soaps and detergents & $12(19.4)$ \\
Cleaning solutions and disinfectants & $9(14.5)$ \\
Laundry detergent pod & $9(14.5)$ \\
Sodium hypochlorite $\leq 6 \%_{\text {Miscellaneous }^{\mathrm{a}}}$ & $9(14.5)$ \\
Class total & $23(37.1)$ \\
\hline
\end{tabular}

${ }^{a}$ Includes ammonia $<10 \%$, car wax, carpet cleaner, deodorants/antiperspirants, dishwasher detergent, dishwater detergent, drain cleaner (irritant), hair product, hand sanitizer unspecified, mouthwash, paint, perfume, shaving cream, talc, toothpaste, and windshield washer fluid

\section{Anticoagulants}

Table S7 details anticoagulant class exposures. Warfarin was the most commonly reported agent (43.2\%) followed by apixaban $(21.6 \%)$ and rivaroxaban (18.9\%).

\section{Chemotherapeutic and Immunological Agents}

Table S8 describes chemotherapeutic and immunological agents. Methotrexate (25.7\%), colchicine (17.1\%), and hydroxychloroquine (14.3\%) were the three most commonly reported agents. Relative methotrexate exposures increased compared to those of $2018 .^{3}$

\section{Anesthetics}

Table S9 describes the 28 anesthetic class exposures. Lidocaine $(25.0 \%)$ was the most commonly reported agent.

\section{Endocrine}

Table S10 describes the 24 endocrine agents reported. Levothyroxine was the most frequently reported agent in this class $(41.7 \%)$ followed by prednisone $(25.0 \%)$.

Table 28 Gases, irritants, vapors, and dusts.

\begin{tabular}{ll}
\hline & $N(\%)$ \\
\hline Carbon monoxide & $37(49.3)$ \\
Smoke & $7(9.3)$ \\
Natural gas & $5(4.5)$ \\
Gases/vapors/irritants/dusts unspecified & $5(6.7)$ \\
Miscellaneous $^{\mathrm{a}}$ & $21(28.0)$ \\
Class total & $75(100)$
\end{tabular}

${ }^{a}$ Includes chlorine, hydrogen sulfide, radon, acetonitrile, carbon dioxide, skunk spray, petroleum vapors, ethylene oxide, sulfur dioxide, cyanide, dust, nitrogen oxides, chloramine, duster (canned air), fumes/vapors/gases unspecified, and polyurethane vapors
Table 29 Plants and fungi.

\begin{tabular}{|c|c|}
\hline & $N(\%)$ \\
\hline Mold & $32(47.8)$ \\
\hline Mitragyna speciosa (kratom) & $11(16.4)$ \\
\hline Miscellaneous $^{\mathrm{a}}$ & $24(35.8)$ \\
\hline Class total & $67(100)$ \\
\hline \multicolumn{2}{|c|}{$\begin{array}{l}\text { a Includes mushroom, other/unknown, Ricinus communis (castor beans), } \\
\text { mushroom, psilocybin, mushroom, Hericium enrinaceus (lion's mane), } \\
\text { Amanita phalloides, betel nut, citronella (Cymbopogon species, lemon- } \\
\text { grass genus), dandelion, digitalis, morning glory (Ipomoea violacea), } \\
\text { almond (Prunus amygdalus), mushroom, cordyceps (Cordyseps } \\
\text { sinensis), taxus (yew), mushroom, Inonotus obliquus (Chaga), Nerium } \\
\text { oleander, phytolacca (pokeweed), plants and fungi unspecified, skullcap } \\
\text { (scutellaria), and mushroom, Ganoderma lucidum (reishi) }\end{array}$} \\
\hline
\end{tabular}

\section{Other Pharmaceuticals}

Table S11 presents the other pharmaceutical product agent class. The majority of the class $(63.6 \%)$ was made up of infrequently reported miscellaneous agents. Unspecified pharmaceutical products were the most commonly reported agent $(22.7 \%)$.

\section{Other Non-pharmaceuticals}

Table S12 describes the other non-pharmaceutical class. Miscellaneous agents made up $70.0 \%$ of this class.

\section{Anti-Parkinsonism Agents}

Table S13 presents the anti-parkinsonism agent class, containing 9 entries. Reported agents included pramipexole, ropinirole, levodopa/carbidopa, and selegiline.

\section{Pulmonary Agents}

Table S14 describes reported pulmonary agents. Montelukast was the most common agent reported $(71.4 \%)$.

\section{Foreign Bodies}

Table S15 details the 4 foreign body ingestions reported to the Registry in 2019 including water beads, cigarette butts, magnets, and razor blades.

\section{Weapons of Mass Destruction.}

Table S16 describes the 3 agents in the weapons of mass destruction class. Botulinum toxin represented $66.7 \%$ of the class. 


\section{Cholinergics}

Table S17 describes the single cholinergic/parasympathetic agent reported, cholinergic/parasympathetic unspecified.

\section{Chelators}

Table S18 describes the single chelator agent reported, trientine $(100 \%)$.

\section{Clinical Signs and Symptoms}

The clinical signs and symptoms categories describe a diverse range of abnormal clinical findings. In order to be reported as being present, predefined criteria must be met for each category. For example, tachycardia is defined as a heart rate greater than 140 beats per minute. Additionally, each case may report more than one abnormality within a group or across groups. For example, a single case entry may have multiple vital sign abnormalities or may have both a vital sign abnormality and a neurologic abnormality. The percentages for these categories and their individual signs and symptoms are calculated relative to the total number of Registry cases $(N=$ 7177). It is therefore possible for the total to be more than $100 \%$.

\section{Toxidromes}

Table 30 reports the 2044 toxidromes reported to the Registry in 2019. Consistent with previous years, the sedative-hypnotic toxidrome was the most common $(10.7 \%) .^{2-4}$ The next top

Table 30 Toxidromes.

\begin{tabular}{ll}
\hline & $N(\%)^{\mathrm{a}}$ \\
\hline Sedative-hypnotic & $770(10.7)$ \\
Anticholinergic & $437(6.1)$ \\
Sympathomimetic & $289(4.0)$ \\
Serotonin syndrome & $186(2.6)$ \\
Opioid & $184(2.6)$ \\
Alcoholic ketoacidosis & $71(1.0)$ \\
Sympatholytic & $53(0.7)$ \\
Washout syndrome & $25(0.3)$ \\
Cholinergic & $10(0.1)$ \\
NMS & $7(0.1)$ \\
Anticonvulsant hypersensitivity & $5(<0.1)$ \\
Miscellaneous & $7(0.1)$ \\
Total & $2044(28.5)$ \\
\hline
\end{tabular}

NMS neuroleptic malignant syndrome

${ }^{\text {a }}$ Percentage based on the total number of cases reported to the Registry in $2019(N=7177)$

${ }^{\mathrm{b}}$ Includes overlap syndromes and cannabinoid hyperemesis four toxidromes were also unchanged from 2018: anticholinergic $(6.1 \%)$, sympathomimetic $(4.0 \%)$, opioid $(2.6 \%)$, and serotonin syndrome $(2.6 \%){ }^{3}$

\section{Major Vital Sign Abnormalities}

Table 31 presents the 1820 vital sign abnormalities reported to the Registry in 2019. Trends were similar to those of previous years. $^{2-4}$ Tachycardia $(11.1 \%)$, hypotension $(5.8 \%)$, and bradycardia $(3.7 \%)$ were the most common vital sign abnormalities reported.

\section{Clinical Signs and Symptoms-Neurologic}

Table 32 describes the 5284 neurologic clinical signs and symptoms reported to the Registry in 2019. Coma/CNS depression $(27.6 \%)$, agitation (15.6\%), and delirium/toxic psychosis $(10.8 \%)$ remained the most commonly reported signs. ${ }^{3}$

\section{Clinical Signs and Symptoms-Cardiovascular and Pulmonary}

Table 33 presents the 610 cardiovascular and 890 pulmonary clinical signs reported to the Registry in 2019. QTc prolongation $(5.1 \%)$ and respiratory depression $(8.3 \%)$ remained the most common signs in their respective categories this year. ${ }^{3}$

\section{Clinical Signs-Other Organ Systems}

Table 34 presents the other organ system clinical signs which include metabolic, renal and musculoskeletal, hematological, gastrointestinal and hepatic, and dermatological. Metabolic abnormalities were again the most frequently reported, and among these, an elevated anion gap (4.2\%) and metabolic acidosis $(3.7 \%)$ were the most common. ${ }^{3}$ Renal and

Table 31 Major vital sign abnormalities.

\begin{tabular}{ll}
\hline & $N(\%)^{\mathrm{a}}$ \\
\hline Tachycardia (HR > 140) & $794(11.1)$ \\
Hypotension (systolic BP <80 mmHg) & $416(5.8)$ \\
Bradycardia $(\mathrm{HR}<50)$ & $266(3.7)$ \\
Bradypnea $(\mathrm{RR}<10)$ & $160(2.2)$ \\
Hypertension (systolic BP $>200 \mathrm{mmHg}$ and/or diastolic & $151(2.1)$ \\
$\quad$ BP $>120 \mathrm{mmHg})$ & $33(0.5)$ \\
Hyperthermia (temp $\left.>105^{\circ} \mathrm{F}\right)$ & $1820(25.4)$ \\
Total &
\end{tabular}

$H R$ heart rate, $B P$ blood pressure, $R R$ respiratory rate

${ }^{a}$ Percentage based on the total number of cases reported to the Registry in $2019(N=7177)$. There were 1521 unique cases $(21.2 \%$ of all Registry cases) reporting at least one major vital sign abnormality. Cases may be associated with more than one major vital sign abnormality 
Table 32 Clinical signs - neurologic.

\begin{tabular}{ll}
\hline & $N(\%)^{\mathrm{a}}$ \\
\hline Coma/CNS depression & $1981(27.6)$ \\
Agitation & $1123(15.6)$ \\
Delirium/toxic psychosis & $776(10.8)$ \\
Hyperflexia/myoclonus/clonus/tremor & $533(7.4)$ \\
Seizures & $398(5.5)$ \\
Hallucinations & $251(3.5)$ \\
Numbness/paresthesia & $78(1.1)$ \\
EPS/dystonia/rigidity & $66(0.9)$ \\
Weakness/paralysis & $63(0.9)$ \\
Peripheral neuropathy (objective) & $15(0.2)$ \\
Total & $5284(73.6)$
\end{tabular}

${ }^{\text {a }}$ Percentage based on the total number of cases reported to the Registry in $2019(N=7177)$; 3685 Registry cases $(51.3 \%)$ reported at least one neurologic clinical effect. Cases may have reported multiple effects

musculoskeletal abnormalities were the next most commonly reported, with acute kidney injury (4.3\%) and rhabdomyolysis $(3.4 \%)$ reported with similar frequencies. Coagulopathy was the most commonly reported hematological abnormality $(2.0 \%)$. Hepatotoxicity was the most common gastrointestinal and hepatic abnormality (2.8\%). Other gastrointestinal and hepatic abnormalities were less common, each reported in less than $1 \%$ of total Registry cases. Among cases reporting any clinical sign, dermatological abnormalities were less frequently reported, with rash being the most commonly reported sign among these (1.6\%).

Table 33 Clinical signs — cardiovascular and pulmonary.

\begin{tabular}{ll}
\hline & $N(\%)^{\mathrm{a}}$ \\
\hline Cardiovascular & \\
Prolonged QTc $(\geq 500 \mathrm{~ms})$ & $364(5.1)$ \\
Prolonged QRS $(\geq 120 \mathrm{~ms})$ & $105(1.5)$ \\
Myocardial injury or infarction & $64(1.4)$ \\
Ventricular dysrhythmia & $55(0.8)$ \\
AV block (>1st degree) & $22(0.3)$ \\
Total & $610(8.5)$ \\
Pulmonary & \\
Respiratory depression & $597(8.3)$ \\
Aspiration pneumonitis & $130(1.8)$ \\
Acute lung injury/ARDS & $117(1.6)$ \\
Asthma/reactive airway disease & $46(0.6)$ \\
Total & $890(12.4)$ \\
\hline
\end{tabular}

$A R D S$ acute respiratory distress syndrome

${ }^{\text {a }}$ Percentage based on the total number of cases reported to the Registry in $2019(N=7177)$; 1291 Registry cases $(18.0 \%)$ reported at least one cardiac or pulmonary clinical effect. Cases may be associated with more than one sign or symptom

\section{Fatalities}

Tables 35 and 36 present cases in which fatalities were reported in 2019. Table 35 includes cases in which a single agent was reported; Table 36 includes cases involving multiple agents. Table S19 in the Supplementary materials presents those fatalities in which it is unknown whether there was a related toxicologic exposure.

There were 91 fatalities in 2019, comprising $1.3 \%$ of Registry cases. Forty-seven cases involved single-agent exposures, 23 involved multiple agents, and in 21 cases it was unknown if there was a toxicologic exposure. There were 28 fatalities in cases of self-harm (1.1\%), a percentage similar to that of the general Registry.

There were 18 fatalities (19.8\%) involving opioids, a decrease from 2018 in which opioids were reported in $34.0 \%$ of

Table 34 Clinical signs - other organ systems.

\begin{tabular}{ll}
\hline & $N(\%)^{\mathrm{a}}$ \\
\hline Metabolic & $714(10.0)$ \\
Elevated anion gap $(>20)$ & $299(4.2)$ \\
Metabolic acidosis $(\mathrm{pH}<7.2)$ & $268(3.7)$ \\
Hypoglycemia $($ glucose $<50 \mathrm{mg} / \mathrm{dL})$ & $113(1.6)$ \\
Elevated osmole gap $(>20)$ & $34(0.5)$ \\
Renal/musculoskeletal & $549(7.6)$ \\
Acute kidney injury $($ creatinine $>2.0 \mathrm{mg} / \mathrm{dL})$ & $308(4.3)$ \\
Rhabdomyolysis $(\mathrm{CPK}>1000 \mathrm{IU} / \mathrm{L})$ & $241(3.4)$ \\
Hematological & $397(5.5)$ \\
Coagulopathy $(\mathrm{PT}>15 \mathrm{~s})$ & $141(2.0)$ \\
Leukocytosis $(\mathrm{WBC}>20 \mathrm{~K} / \mu \mathrm{L})$ & $90(1.3)$ \\
Thrombocytopenia $(\mathrm{platelets}<100 \mathrm{~K} / \mu \mathrm{L})$ & $79(1.1)$ \\
Hemolysis $(\mathrm{Hgb}<10 \mathrm{~g} / \mathrm{dL})$ & $59(0.8)$ \\
Methemoglobinemia $(\mathrm{MetHgb} \geq 2 \%)$ & $19(0.3)$ \\
Pancytopenia & $9(0.1)$ \\
Gastrointestinal/hepatic & $323(4.5)$ \\
Hepatotoxicity $(\mathrm{AST} \geq 1000 \mathrm{IU} / \mathrm{L})$ & $199(2.8)$ \\
Pancreatitis & $54(0.8)$ \\
Gastrointestinal bleeding & $45(0.6)$ \\
Corrosive injury & $22(0.3)$ \\
Intestinal ischemia & $3(0.0)$ \\
Dermatological & $230(3.2)$ \\
Rash & $115(1.6)$ \\
Blister/bullae & $75(1.0)$ \\
Necrosis & $31(0.4)$ \\
Angioedema & $9(0.1)$ \\
\hline
\end{tabular}

$C P K$ creatine phosphokinase, $P T$ prothrombin time, $W B C$ white blood cells, $A S T$ aspartate aminotransferase

${ }^{a}$ Percentage equals the number of cases reporting specific clinical signs compared to the total number of Registry cases in $2019(N=7177)$. Cases may be associated with more than one sign or symptom 
Table 352019 fatalities reported in ToxIC Registry with known toxicological exposure: single agent.

\begin{tabular}{|c|c|c|c|c|c|}
\hline $\begin{array}{l}\text { Age } \\
\text { /gender }^{\mathrm{a}}\end{array}$ & Agents involved & Clinical findings & $\begin{array}{l}\text { Life } \\
\text { support } \\
\text { withdrawn }\end{array}$ & $\begin{array}{l}\text { Brain } \\
\text { death } \\
\text { confirmed }\end{array}$ & Treatment $^{\mathrm{b}}$ \\
\hline $14 \mathrm{~F}$ & Acetaminophen & HPT & Unknown & & NAC \\
\hline $15 \mathrm{~F}$ & Acetaminophen & None & No & & NAC \\
\hline $32 \mathrm{~F}$ & Acetaminophen & DLM, HGY, MA, HPT, CPT & Unknown & & Vitamin $\mathrm{K}$ \\
\hline $36 \mathrm{~F}$ & Acetaminophen & $\begin{array}{l}\text { HT, CNS, DLM, HGY, MA, } \\
\text { AG, HPT, GIB, PLT, AKI }\end{array}$ & Yes & No & $\begin{array}{l}\text { Folate, NAC, thiamine, continuous renal replacement, } \\
\text { intubation, IV fluids }\end{array}$ \\
\hline $37 \mathrm{~F}$ & Acetaminophen & $\begin{array}{l}\text { HT, TC, HY, QTC, ALI, CNS, } \\
\text { MA, HPT, AKI }\end{array}$ & Yes & No & $\begin{array}{l}\mathrm{NAC}, \mathrm{NaHCO}_{3} \text {, vasopressors (norepinephrine, } \\
\text { vasopressin), glucose }>5 \% \text {, intubation, IV fluids }\end{array}$ \\
\hline $46 \mathrm{~F}$ & Acetaminophen & $\begin{array}{l}\text { TC, QTC, ALI, CNS, MA, AG, } \\
\text { HPT, GII, CPT, PLT, WBC, } \\
\text { AKI }\end{array}$ & No & & $\begin{array}{l}\mathrm{NAC}, \mathrm{NaHCO}_{3} \text {, vitamin } \mathrm{K} \text {, vasopressors } \\
\text { (norepinephrine), beta-blockers, opioids, } \mathrm{CPR} \text {, } \\
\text { intubation, IV fluids }\end{array}$ \\
\hline $46 \mathrm{~F}$ & Acetaminophen & HT, AP, RD, CNS, HPT & Unknown & & $\begin{array}{l}\text { NAC, vasopressors (norepinephrine, vasopressin), } \\
\text { intubation, IV fluids }\end{array}$ \\
\hline $48 \mathrm{M}$ & Acetaminophen & $\begin{array}{l}\text { HYT, RD, MA, AG, HPT, CPT, } \\
\text { PLT, WBC, AKI }\end{array}$ & No & & NAC, CPR, intubation, IV fluids \\
\hline $51 \mathrm{M}$ & Acetaminophen & $\begin{array}{l}\text { RD, CNS, MA, AG, HPT, CPT, } \\
\text { AKI }\end{array}$ & Yes & No & $\begin{array}{l}\text { NAC, vasopressors (epinephrine, vasopressin), } \\
\text { continuous renal replacement }\end{array}$ \\
\hline $67 \mathrm{~F}$ & Acetaminophen & PNC & No & & NAC \\
\hline $72 \mathrm{M}$ & Acetaminophen & WBC & Unknown & & NAC, opioids, IV fluids \\
\hline $77 \mathrm{~F}$ & Acetaminophen & HT, CNS, MA, HPT, AKI & No & & $\begin{array}{l}\text { NAC, vasopressors (norepinephrine), continuous renal } \\
\text { replacement, intubation, IV fluids }\end{array}$ \\
\hline$>89 \mathrm{~F}$ & Acetaminophen & $\begin{array}{l}\text { Sedative-hypnotic toxidrome, } \\
\text { CNS, HPT }\end{array}$ & No & & NAC \\
\hline $58 \mathrm{~F}$ & Antimony & $\mathrm{SZ}$ & Yes & Yes & None \\
\hline $4 \mathrm{M}$ & Baclofen & None & No & & IV fluids \\
\hline $18 \mathrm{~F}$ & Benzonatate & HT, HY, CNS, MA, AG & Yes & Yes & $\begin{array}{l}\text { Naloxone/nalmefene, anticonvulsants, benzodiazepines, } \\
\text { CPR, intubation }\end{array}$ \\
\hline$>89 \mathrm{~F}$ & Carpet cleaner & HT, MI, RD, MA, HYS, CPT & No & & Intubation \\
\hline $14 \mathrm{moF}$ & Cocaine & $\begin{array}{l}\text { Sympathomimetic syndrome, } \\
\text { HT, TC, VD, QRS, QTC, RD, } \\
\text { CNS, SZ, MA, AG, CPT }\end{array}$ & Yes & Yes & $\begin{array}{l}\mathrm{NaHCO}_{3} \text {, vasopressors (epinephrine), anticonvulsants, } \\
\text { CPR, intubation, IV fluids }\end{array}$ \\
\hline $33 \mathrm{M}$ & Cocaine & $\begin{array}{l}\text { HT, MI, ALI, CNS, SZ, MA, } \\
\text { AKI }\end{array}$ & Yes & No & None \\
\hline $15 \mathrm{M}$ & Diphenhydramine & $\begin{array}{l}\text { TC, VD, QRS, CNS, DLM, SZ, } \\
\text { MA, AG, WBC, RBM }\end{array}$ & No & & $\begin{array}{l}\mathrm{NaHCO}_{3} \text {, vasopressors (norepinephrine), } \\
\text { benzodiazepines, opioids, CPR, intubation, IV fluids }\end{array}$ \\
\hline $15 \mathrm{~F}$ & Diphenhydramine & $\begin{array}{l}\text { Anticholinergic toxidrome, } \\
\text { HTN, TC, QTC, CNS, MA, } \\
\text { AG }\end{array}$ & Yes & Yes & Activated charcoal \\
\hline $17 \mathrm{~F}$ & Ethanol & $\begin{array}{l}\text { Sedative-hypnotic toxidrome, } \\
\text { HT, BP, AP, RD, CNS, MA, } \\
\text { AG }\end{array}$ & Yes & Yes & $\mathrm{CPR}$, intubation, IV fluids \\
\hline $65 \mathrm{M}$ & Ethanol & DLM & Yes & No & Benzodiazepines, intubation, IV fluids \\
\hline $20 \mathrm{M}$ & Fentanyl & $\begin{array}{l}\text { Opioid toxidrome, HT, BP, VD, } \\
\text { MI, ALI, RD, CNS, MA, AG, } \\
\text { AKI }\end{array}$ & Unknown & & $\begin{array}{l}\mathrm{NaHCO}_{3} \text {, vasopressors (epinephrine, norepinephrine), } \\
\text { continuous renal replacement, } \mathrm{CPR} \text {, intubation, IV } \\
\text { fluids }\end{array}$ \\
\hline $21 \mathrm{M}$ & Fentanyl & $\begin{array}{l}\text { HT, VD, MI, AP, RD, CNS, } \\
\text { MA, AG, HPT, GII, WBC, } \\
\text { AKI }\end{array}$ & No & & $\begin{array}{l}\mathrm{NAC}, \mathrm{NaHCO}_{3} \text {, vasopressors (epinephrine, } \\
\text { norepinephrine, vasopressin), antiarrhythmics, } \\
\text { benzodiazepines, neuromuscular blockers, CPR, } \\
\text { cardioversion, intubation }\end{array}$ \\
\hline $34 \mathrm{~F}$ & Fentanyl & $\begin{array}{l}\text { Opioid toxidrome, HT, BP, MI, } \\
\text { ALI, RD, CNS, MA, HPT, } \\
\text { AKI }\end{array}$ & Yes & No & $\begin{array}{l}\text { Flumazenil, naloxone/nalmefene, vasopressors } \\
\text { (epinephrine, norepinephrine), CPR, intubation, IV } \\
\text { fluids }\end{array}$ \\
\hline $50 \mathrm{M}$ & Fentanyl & $\begin{array}{l}\text { Opioid toxidrome, BP, RD, } \\
\text { CNS, HPT, AKI, RBM }\end{array}$ & Yes & No & $\begin{array}{l}\text { NAC, naloxone/nalmefene, vasopressors (phenylephrine), } \\
\text { antihypertensives, benzodiazepines, beta blockers, } \\
\text { opioids, hemodialysis, intubation, IV fluids }\end{array}$ \\
\hline
\end{tabular}


Table 35 (continued)

\begin{tabular}{|c|c|c|c|c|c|}
\hline $\begin{array}{l}\text { Age } \\
\text { /gender }^{\mathrm{a}}\end{array}$ & Agents involved & Clinical findings & $\begin{array}{l}\text { Life } \\
\text { support } \\
\text { withdrawn }\end{array}$ & $\begin{array}{l}\text { Brain } \\
\text { death } \\
\text { confirmed }\end{array}$ & Treatment $^{\mathrm{b}}$ \\
\hline $18 \mathrm{M}$ & Flecainide & HT, BC, QRS, QTC, CNS & No & & $\begin{array}{l}\mathrm{NaHCO}_{3} \text {, vasopressors (epinephrine), antiarrhythmics, } \\
\text { steroids, CPR, intubation, IV fluids }\end{array}$ \\
\hline $27 \mathrm{~F}$ & Heroin & $\begin{array}{l}\text { Sedative-hypnotic toxidrome, } \\
\text { AKI }\end{array}$ & No & & $\begin{array}{l}\text { Lipid therapy, NAC, naloxone/nalmefene, vasopressors, } \\
\text { CPR, intubation, IV fluids }\end{array}$ \\
\hline $31 \mathrm{M}$ & Heroin & $\mathrm{VD}, \mathrm{RD}, \mathrm{CNS}$ & Yes & Yes & $\begin{array}{l}\text { Naloxone/nalmefene, anticonvulsants, benzodiazepines, } \\
\text { neuromuscular blockers, opioids, CPR, intubation, IV } \\
\text { fluids }\end{array}$ \\
\hline $39 \mathrm{M}$ & Heroin & $\begin{array}{l}\text { HTN, QRS, QTC, RD, CNS, } \\
\text { MA, PNC }\end{array}$ & Yes & Yes & $\begin{array}{l}\text { Naloxone/nalmefene, vasopressors (epinephrine), } \\
\text { benzodiazepines, opioids, intubation, IV fluids }\end{array}$ \\
\hline $41 \mathrm{~F}$ & Heroin & $\begin{array}{l}\text { Opioid toxidrome, AP, CNS, } \\
\text { RFX }\end{array}$ & Yes & Yes & None \\
\hline $60 \mathrm{~F}$ & Heroin & $\begin{array}{l}\text { HTN, BP, VD, MI, RD, CNS, } \\
\text { MA, AG, WBC }\end{array}$ & No & & $\begin{array}{l}\text { Naloxone/nalmefene, nitrites, } \mathrm{NaHCO}_{3} \text {, bronchodilators } \\
\text { antihypertensives, benzodiazepines, opioids, IV fluids }\end{array}$ \\
\hline $13 \mathrm{M}$ & $\begin{array}{l}\text { Loxosceles } \\
\text { (recluse spider) }\end{array}$ & RS & No & & None \\
\hline $40 \mathrm{M}$ & Metformin & $\mathrm{HT}, \mathrm{RD}, \mathrm{CNS}, \mathrm{MA}, \mathrm{AG}$ & No & & $\begin{array}{l}\mathrm{NaHCO}_{3} \text {, vasopressors (epinephrine, norepinephrine, } \\
\text { vasopressin), hemodialysis, CPR, intubation, IV fluids }\end{array}$ \\
\hline $48 \mathrm{M}$ & Metformin & $\begin{array}{l}\text { HT, TC, AGT, MA, AG, GIB, } \\
\text { WBC, AKI }\end{array}$ & No & & $\begin{array}{l}\text { Calcium, } \mathrm{NaHCO}_{3} \text {, vasopressors (norepinephrine), } \\
\text { hemodialysis, continuous renal replacement, CPR, } \\
\text { intubation, IV fluids }\end{array}$ \\
\hline $55 \mathrm{M}$ & Methanol & $\begin{array}{l}\text { Sedative-hypnotic toxidrome, } \\
\text { HGY, AG, OG, AKI }\end{array}$ & Yes & Yes & $\begin{array}{l}\text { Fomepizole, vasopressors (epinephrine, norepinephrine) } \\
\text { continuous renal replacement, CPR, IV fluids }\end{array}$ \\
\hline $85 \mathrm{~F}$ & Methotrexate & HT, CNS, PLT, PCT & Yes & No & Folate, benzodiazepines, steroids \\
\hline$>89 \mathrm{~F}$ & Methotrexate & DLM, HYS, CPT, PLT, RS, BL & No & & Folate, vasopressors (norepinephrine), IV fluids \\
\hline $16 \mathrm{~F}$ & Oxycodone & $\begin{array}{l}\text { Opioid toxidrome, HT, VD, RD, } \\
\text { CNS, MA, HPT, WBC }\end{array}$ & Yes & Yes & $\begin{array}{l}\text { Naloxone/nalmefene, vasopressors (norepinephrine), } \\
\text { intubation, IV fluids }\end{array}$ \\
\hline $65 \mathrm{~F}$ & Phenytoin & HYT, SZ, WBC & Yes & Yes & MDAC \\
\hline $61 \mathrm{~F}$ & Quetiapine & $\begin{array}{l}\text { Sedative-hypnotic toxidrome, } \\
\text { HT, VD, RD }\end{array}$ & Yes & No & $\begin{array}{l}\text { Lipid therapy, vasopressors (epinephrine), intubation, IV } \\
\text { fluids }\end{array}$ \\
\hline $69 \mathrm{~F}$ & Salicylic acid & $\mathrm{TC}$ & No & & $\mathrm{NaHCO}_{3}$, IV fluids \\
\hline $43 \mathrm{~F}$ & Smoke & $\mathrm{RAD}, \mathrm{CNS}$ & Yes & Yes & Hydroxocobalamin, HBO, intubation, IV fluids \\
\hline $21 \mathrm{M}$ & Tetrahydrocannabinol & ALI, CNS, AKI & No & & $\begin{array}{l}\text { Vasopressors (norepinephrine, vasopressin, dopamine), } \\
\text { antiarrhythmics, opioids, steroids, continuous renal } \\
\text { replacement, ECMO, intubation, IV fluids }\end{array}$ \\
\hline $33 \mathrm{~F}$ & Verapamil & $\begin{array}{l}\text { Sedative-hypnotic toxidrome, } \\
\text { HT, BC, AVB, RAD, CNS, } \\
\text { MA, AG, CPT, AKI }\end{array}$ & Yes & No & $\begin{array}{l}\text { HIE, vasopressors (epinephrine, norepinephrine, } \\
\text { vasopressin), activated charcoal, continuous renal } \\
\text { replacement, intubation, IV fluids }\end{array}$ \\
\hline $47 \mathrm{~F}$ & Verapamil & $\begin{array}{l}\text { HT, BC, VD, QRS, QTC, AVB, } \\
\text { ALI, AP, RD, CNS, MA, AKI }\end{array}$ & No & & $\begin{array}{l}\text { Calcium, glucagon, } \mathrm{HIE} \text {, lipid therapy, } \mathrm{NaHCO}_{3}, \\
\text { vasopressors (epinephrine, norepinephrine, } \\
\text { vasopressin, dopamine), glucose }>5 \% \text {, intubation, IV } \\
\text { fluids, pacemaker }\end{array}$ \\
\hline
\end{tabular}

Based on the response from the medical toxicologist "Did the patient have a toxicological exposure?" equals yes with known agent(s)

$A G$ anion gap, $A G T$ agitation, $A K I$ acute kidney injury, $A L I$ acute lung injury/ARDS, $A P$ aspiration pneumonitis, $A V B$ AV block, $B C$ bradycardia, $B L$ blisters/bullae, $B P$ bradypnea, $C N S$ coma/CNS depression, $C P T$ coagulopathy, $C R V$ corrosive injury, DLM delirium, GIB GI bleeding, GII intestinal ischemia, $H G Y$ hypoglycemia, HPT hepatoxicity, $H T$ hypotension, $H T N$ hypertension, $H Y S$ hemolysis, $H Y$ hypothermia, $H Y T$ hyperthermia, $M A$ metabolic acidosis, $M I$ myocardial injury/ischemia, $O G$ osmolar gap, $P C T$ pancytopenia, $P L T$ thrombocytopenia, $P N C$ pancreatitis, $Q T C$ QTc prolongation, $R A D$ asthma/reactive airway disease, $R B M$ rhabdomyolysis, $R D$ respiratory depression, $R F X$ hyperreflexia/tremor, $R S$ rash, $S Z$ seizures, $T C$ tachycardia, $V D$ ventricular dysrhythmia, $W B C$ leukocytosis, $C P R$ cardiopulmonary resuscitation, $E C M O$ extracorporeal membrane oxygenation, $H B O$ hyperbaric oxygenation, $\mathrm{HIE}$ high-dose insulin euglycemic therapy, $\mathrm{MDAC}$ multiple-dose activated charcoal, $\mathrm{NAC}$ n-acetyl cysteine, $\mathrm{NaHCO}_{3}$ sodium bicarbonate

${ }^{\text {a }}$ Age in years unless otherwise stated

${ }^{\mathrm{b}}$ Pharmacological and non-pharmacological support as reported by medical toxicologist 
Table 362019 fatalities reported in ToxIC Registry with known toxicological exposure: multiple agents.

\begin{tabular}{|c|c|c|c|c|c|}
\hline $\begin{array}{l}\text { Age/ } \\
\text { gender }^{\mathrm{a}}\end{array}$ & Agents involved & Clinical findings & $\begin{array}{l}\text { Life } \\
\text { support } \\
\text { withdrawn }\end{array}$ & $\begin{array}{l}\text { Brain } \\
\text { death } \\
\text { confirmed }\end{array}$ & Treatment $^{\mathrm{b}}$ \\
\hline $15 \mathrm{~F}$ & $\begin{array}{l}\text { Acetaminophen, } \\
\text { caffeine }\end{array}$ & $\mathrm{V}$ & Unknown & & NAC \\
\hline $16 \mathrm{~F}$ & $\begin{array}{l}\text { Acetaminophen, aspirin, } \\
\text { caffeine }\end{array}$ & None & No & & None \\
\hline $17 \mathrm{M}$ & $\begin{array}{l}\text { Acetaminophen, } \\
\text { anticholinergic } \\
\text { unspecified }\end{array}$ & $\begin{array}{l}\text { Anticholinergic toxidrome, } \\
\text { AGT, V }\end{array}$ & No & & NAC, IV fluids \\
\hline $61 \mathrm{M}$ & $\begin{array}{l}\text { Acetaminophen, opioid } \\
\text { unspecified }\end{array}$ & $\begin{array}{l}\text { HT, MI, RD, CNS, MA, AG, } \\
\text { GIB, AKI }\end{array}$ & No & & $\begin{array}{l}\text { NAC, vasopressors (norepinephrine), intubation, IV } \\
\text { fluids }\end{array}$ \\
\hline $75 \mathrm{M}$ & $\begin{array}{l}\text { Acetaminophen, } \\
\text { donepezil, } \\
\text { levothyroxine, } \\
\text { lisinopril, tamsulosin }\end{array}$ & $\mathrm{HT}, \mathrm{BC}, \mathrm{RD}$ & No & & $\begin{array}{l}\text { Glucagon, methylene blue, NAC, vasopressors } \\
\text { (epinephrine, norepinephrine, vasopressin, } \\
\text { phenylephrine), continuous renal replacement therapy, } \\
\text { intubation }\end{array}$ \\
\hline $79 \mathrm{~F}$ & $\begin{array}{l}\text { Acetaminophen, } \\
\text { codeine }\end{array}$ & RD, CNS, MA, HPT, PNC & Yes & Yes & NAC, vasopressors (dobutamine) \\
\hline $60 \mathrm{~F}$ & $\begin{array}{l}\text { Acetylsalicylic acid, } \\
\text { ethanol }\end{array}$ & $\begin{array}{l}\text { HT, TC, BC, QRS, RD, CNS, } \\
\text { SZ, MA, AG, HPT, CPT, } \\
\text { RBM }\end{array}$ & No & & $\begin{array}{l}\text { Calcium, Fab for digoxin, methylene blue, } \\
\text { naloxone/nalmefene, } \mathrm{NaHCO}_{3} \text {, vitamin } \mathrm{K} \text {, } \\
\text { vasopressors, antiarrhythmics, benzodiazepines, } \\
\text { glucose }>5 \% \text {, steroids, activated charcoal, } \\
\text { hemodialysis, intubation }\end{array}$ \\
\hline $49 \mathrm{M}$ & $\begin{array}{l}\text { Amlodipine, } \\
\text { nortriptyline, } \\
\text { duloxetine, } \\
\text { meloxicam }\end{array}$ & $\mathrm{HT}, \mathrm{RD}, \mathrm{CNS}, \mathrm{MA}, \mathrm{AKI}$ & Yes & Unknown & $\begin{array}{l}\text { Calcium, glucagon, HIE, lipid therapy, methylene blue, } \\
\text { vasopressors (epinephrine, norepinephrine, } \\
\text { vasopressin, phenylephrine, dopamine, dobutamine), } \\
\text { benzodiazepines, neuromuscular blockers, opioids, } \\
\text { intubation, IV fluids }\end{array}$ \\
\hline $67 \mathrm{M}$ & Amlodipine, lorazepam & $\begin{array}{l}\text { BC, QRS, RD, CNS, MA, AG, } \\
\text { CPT, AKI, RBM }\end{array}$ & Yes & Yes & $\begin{array}{l}\text { Atropine, calcium, glucagon, } \mathrm{HIE}, \mathrm{NaHCO}_{3} \text {, } \\
\text { vasopressors (epinephrine, norepinephrine), } \\
\text { benzodiazepines, glucose }>5 \% \text {, neuromuscular } \\
\text { blockers, intubation, IV fluids }\end{array}$ \\
\hline $70 \mathrm{M}$ & $\begin{array}{l}\text { Amlodipine, aspirin, } \\
\text { fluoxetine }\end{array}$ & HT, ALI, CNS, DLM, AKI & No & & $\begin{array}{l}\text { Calcium, glucagon, hydroxocobalamin, } \mathrm{HIE} \text {, lipid } \\
\text { therapy, methylene blue, } \mathrm{NaHCO}_{3} \text {, hemodialysis, } \\
\text { continuous renal replacement therapy, IV fluids }\end{array}$ \\
\hline $68 \mathrm{M}$ & $\begin{array}{l}\text { Baclofen, clonidine, } \\
\text { hydromorphone, } \\
\text { bupivacaine }\end{array}$ & $\begin{array}{l}\text { Sedative-hypnotic toxidrome, } \\
\text { CNS, WBC, AKI }\end{array}$ & Yes & Yes & IV fluids, opioids \\
\hline $31 \mathrm{M}$ & Cocaine, ethanol & AGT, DLM & Unknown & & IV fluids \\
\hline $37 \mathrm{M}$ & $\begin{array}{l}\text { Cocaine, opioid } \\
\text { unspecified }\end{array}$ & $\begin{array}{l}\text { HTN, HT, BP, QTC, AP, RD, } \\
\text { CNS, MA, AG, HPT, WBC, } \\
\text { RBM }\end{array}$ & Yes & Yes & $\begin{array}{l}\text { Vasopressors (norepinephrine, vasopressin), intubation, } \\
\text { IV fluids }\end{array}$ \\
\hline $83 \mathrm{~F}$ & $\begin{array}{l}\text { Digoxin, diltiazem, } \\
\text { metoprolol }\end{array}$ & $\mathrm{HT}, \mathrm{BC}, \mathrm{CNS}$ & Yes & Unknown & $\begin{array}{l}\text { Fab for digoxin, glucagon, vasopressors (dopamine), IV } \\
\text { fluids }\end{array}$ \\
\hline $58 \mathrm{~F}$ & Ethanol, valacyclovir & $\begin{array}{l}\text { HTN, HT, QTC, CNS, SZ, } \\
\text { MA, AKI }\end{array}$ & Yes & Yes & $\begin{array}{l}\text { Thiamine, vasopressors (norepinephrine), } \\
\text { antihypertensives, benzodiazepines, opioids, } \\
\text { vasodilators, intubation, IV fluids }\end{array}$ \\
\hline $67 \mathrm{~F}$ & Ethanol, alprazolam, & $\begin{array}{l}\text { Serotonin syndrome, BC, ALI, } \\
\text { AGT, HCN, RFX, HYS }\end{array}$ & Yes & Yes & Antipsychotics, benzodiazepines \\
\hline $34 \mathrm{~F}$ & Fentanyl, norfentanyl & $\begin{array}{l}\text { Opioid toxidrome, RD, CNS, } \\
\text { SZ, AG, WBC }\end{array}$ & Yes & Yes & Naloxone/nalmefene, benzodiazepines \\
\hline $66 \mathrm{~F}$ & Glimepiride, tramadol & AGT, HGY & No & & Octreotide, glucose $>5 \%$, IV fluids \\
\hline $87 \mathrm{~F}$ & $\begin{array}{l}\text { Haloperidol, } \\
\text { thiothixene, } \\
\text { aripiprazole }\end{array}$ & EPS & No & & IV fluids \\
\hline $63 \mathrm{~F}$ & $\begin{array}{l}\text { Hydrocodone, tramadol, } \\
\text { ibuprofen }\end{array}$ & $\begin{array}{l}\text { Sedative-hypnotic toxidrome, } \\
\text { HT, ALI, CNS, HPT, RBM }\end{array}$ & Yes & Yes & NAC, naloxone/nalmefene, intubation, IV fluids \\
\hline $21 \mathrm{~F}$ & $\begin{array}{l}\text { Oxycodone, ibuprofen, } \\
\text { aripiprazole, }\end{array}$ & $\begin{array}{l}\text { HT, QRS, MI, ALI, AP, RD, } \\
\text { CNS, HGY, MA, PNC, }\end{array}$ & No & & $\begin{array}{l}\text { Calcium, glucagon, vasopressors (isoproterenol), glucose } \\
>5 \% \text {, intubation, IV fluids }\end{array}$ \\
\hline
\end{tabular}


Table 36 (continued)

\begin{tabular}{|c|c|c|c|c|c|}
\hline $\begin{array}{l}\text { Age/ } \\
\text { gender }^{a}\end{array}$ & Agents involved & Clinical findings & $\begin{array}{l}\text { Life } \\
\text { support } \\
\text { withdrawn }\end{array}$ & $\begin{array}{l}\text { Brain } \\
\text { death } \\
\text { confirmed }\end{array}$ & Treatment $^{\mathrm{b}}$ \\
\hline
\end{tabular}

\begin{tabular}{|c|c|c|c|c|c|}
\hline & fluoxetine, & & & & \\
\hline $65 \mathrm{M}$ & $\begin{array}{l}\text { Tamsulosin, } \\
\text { emtricitabine, } \\
\text { darunavir, } \\
\text { elvitegravir }\end{array}$ & HT & Unknown & & Vasopressors (norepinephrine) \\
\hline $60 \mathrm{M}$ & Ziprasidone, alprazolam & $\begin{array}{l}\text { Sedative-hypnotic toxidrome, } \\
\text { HT, BP, QRS, QTC, RD, } \\
\text { CNS, MA }\end{array}$ & Yes & Yes & $\begin{array}{l}\text { Lipid therapy, vasopressors (dopamine), } \\
\text { CPR, intubation }\end{array}$ \\
\hline
\end{tabular}

Based on response from Medical Toxicologist "Did the patient have a toxicological exposure?" equals yes with known agent(s)

$A G$ anion gap, $A G T$ agitation, $A L I$ acute lung injury/ARDS, $A K I$ acute kidney injury, $A P$ aspiration pneumonitis, $B C$ bradycardia, $B P$ bradypnea, $C N S$ coma/CNS depression, $C P T$ coagulopathy, $C R V$ corrosive injury, $D L M$ delirium, $E P S$ dystonia, GIB GI bleeding, $H C N$ hallucinations, $H G Y$ hypoglycemia, HLS hemolysis, HPT hepatoxicity, $H T$ hypotension, $H T N$ hypertension, $H Y S$ hemolysis, MA metabolic acidosis, $M I$ myocardial infarction, $P L T$ thrombocytopenia, $P N C$ pancreatitis, $Q R S$ QRS prolongation, $Q T C$ QTc prolongation, $R B M$ rhabdomyolysis, $R D$ respiratory depression, $R F X$ hyperreflexia/tremor, $S Z$ seizures, $T C$ tachycardia, $V$ vomiting, $W B C$ leukocytosis, $C P R$ cardiopulmonary resuscitation, $H I E$ high-dose insulin euglycemic therapy, $\mathrm{NAC}$ n-acetyl cysteine, $\mathrm{NaHCO}_{3}$ sodium bicarbonate

${ }^{\text {a }}$ Age listed in years unless otherwise stated

${ }^{\mathrm{b}}$ Pharmacological and non-pharmacological support as reported by medical toxicologist

Registry deaths. ${ }^{3}$ Fentanyl was reported in 5 deaths $(5.5 \%)$ this year compared to $9.4 \%$ in $2018^{3} ; 10$ deaths $(11.0 \%)$ were reported as single-opioid ingestions in 2019.

Acetaminophen was the most common agent involved in both single- and multiple-agent fatalities; there were 19 fatalities $(20.9 \%)$ involving acetaminophen, 13 as a single agent. A single envenomation death was reported after Loxosceles envenomation in a 13 -year-old. A singleagent tetrahydrocannabinol death was reported in a 21 year-old who presented with acute lung injury, acute kidney injury, and CNS depression. He was treated with vasopressors, opioids, antiarrhythmics, steroids, IV fluid resuscitation, endotracheal intubation, continuous renal replacement therapy, and ECMO.

In 2019, there were 14 pediatric (age $0-18$ years) deaths due to a known toxicologic exposure (20.0\%), compared to $13.3 \%$ in 2018 . $^{3}$ The age range was 14 months to 18 years. Eleven were single-agent exposures and 3 involved multiple agents. Five (35.7\%) involved acetaminophen, 2 as single agent ingestions. Only one death in a pediatric patient involved opioids and was reported as a single agent oxycodone ingestion in a 16-year-old. One single agent ethanol death was reported in a 17-year-old. Two deaths in pre-teens $(\leq 12$ years of age) were reported and involved single-agent exposures of baclofen and cocaine in a 4-year-old and 14-month-old, respectively.

There were 44 fatality cases in which life support was withdrawn, representing $0.6 \%$ of Registry cases. It was unknown whether life support was withdrawn in an additional 14 cases. Brain death was declared in 27 cases.

\section{Adverse Drug Reactions}

Table 37 presents the common drugs associated with adverse drug reactions reported to the Registry in 2019. Three hundred two (302) ADRs (4.2\% of Registry cases) were reported in 2019 , representing nearly a doubling from last year $(2.3 \%$ of Registry cases). ${ }^{3}$ Lithium was again the most common drug reported (5.6\%), similar to that of previous years, ${ }^{2-4}$ followed by digoxin $(3.6 \%)$.

\section{Treatment}

\section{Antidotal Therapy}

Table 38 describes the 2410 antidotes reported to the Registry in 2019. Similar to previous years, $\mathrm{N}$-acetylcysteine (31.2\%), followed by naloxone/nalmefene (13.7\%), were the two most common antidotes reported. ${ }^{3,4}$ This year, thiamine advanced to the third most common antidote reported (11.5\%), overtaking sodium bicarbonate $(9.5 \%)$.

\section{Antivenom Therapy}

Table 39 presents data on antivenom therapies reported to the Registry. Crotalidae polyvalent immune fab (ovine) made up the majority (73.9\%) of antivenom administered; however, it was less common than in 2018 (94.2\%). ${ }^{3}$ Anavip Fab2 antivenom was a new antivenom in 2019 and made up 19.9\% of this class. 
Table 37 Most common drugs associated with adverse drug reactions.

\begin{tabular}{lr}
\hline & $N(\%)$ \\
\hline Lithium & $17(5.6)$ \\
Digoxin & $11(3.6)$ \\
Haloperidol & $8(2.6)$ \\
Fentanyl & $8(2.6)$ \\
Metoprolol & $8(2.6)$ \\
Phenytoin & $7(2.3)$ \\
Quetiapine & $7(2.3)$ \\
Metformin & $6(2.0)$ \\
Baclofen & $5(1.7)$ \\
Lidocaine & $5(1.7)$ \\
Tramadol & $5(1.7)$ \\
Glipizide & $5(1.7)$ \\
Valproic acid & $5(1.7)$ \\
Glimepiride & $5(1.7)$ \\
Gabapentin & $5(1.7)$ \\
Acetaminophen & $5(1.7)$ \\
Clonidine & $5(1.7)$ \\
Miscellaneous & \\
Total & $185(61.3)$ \\
\hline
\end{tabular}

${ }^{a}$ Includes escitalopram, morphine, lamotrigine, citalopram, ethanol, alprazolam, fluphenazine, clonazepam, olanzapine, methotrexate, methadone, diphenhydramine, duloxetine, diltiazem, bupropion, venlafaxine, nortriptyline, cariprazine, sertraline, doxylamine, dapsone, carvedilol, lorazepam, benzocaine, lisinopril, aripiprazole, ibuprofen, benztropine, topiramate, amlodipine, verapamil, vortioxetine, ziprasidone, fluoxetine, unknown, nadolol, bupivacaine, prochlorperazine, oxcarbazepine, oxybutynin, oxycodone, cyclobenzaprine, paroxetine, buspirone, ceftriaxone, flecainide, cefazolin, anticholinergic unspecified, clarithromycin, epinephrine, antivenom unspecified, cannabinoid nonsynthetic, amiodarone, digitalis, dofetilide, divalproex, disulfiram, caffeine, atomoxetine, atorvastatin, cocaine, butalbital, crotalus (rattlesnake), diazoxide, dextromethorphan, doxepin, snake unspecified, ipilimumab, pharmaceutical cannabidiol, phenylephrine, pramipexole, prilocaine, propofol, risperidone, ropivacaine, scopolamine, oseltamivir, sevoflurane, opioid unspecified, succinylcholine, sympathomimetic unspecified, thiothixene, timolol, tizanidine, tranylcypromine, trazodone, unspecified pharmaceutical, vitamin B3 (niacin), warfarin, sed-hypnotic/muscle relaxant unspecified, liraglutide, fluvoxamine, herbal (dietary) multibotanical, herbals/dietary supps/vitamins unspecified, hydralazine, hydrochlorothiazide, hydroxyzine, insulin, iodine monochloride, iron, paliperidone, linezolid, fluconazole, lurasidone, magnesium, marijuana, melatonin, meperidine, metronidazole, minocycline, n-acetylcysteine, naltrexone, nivolumab, and zolpidem

\section{Pharmacologic Supportive Care}

Table 40 describes the 3157 pharmacologic supportive care treatments reported in 2019. Benzodiazepines were again the most commonly reported agents $(51.2 \%)^{3}$ followed by opioids (13.0\%) and vasopressors (10.0\%).
Table 38 Antidotal therapy.

\begin{tabular}{ll}
\hline & $N(\%)^{\mathrm{a}}$ \\
\hline$N$-Acetylcysteine & $751(31.2)$ \\
Naloxone/nalmefene & $331(13.7)$ \\
Thiamine & $276(11.5)$ \\
Sodium bicarbonate & $230(9.5)$ \\
Folate & $192(8.0)$ \\
Fomepizole & $85(3.5)$ \\
Physostigmine & $77(3.2)$ \\
Glucagon & $73(3.0)$ \\
Calcium & $64(2.7)$ \\
Atropine & $44(1.8)$ \\
Flumazenil & $36(1.5)$ \\
Insulin-euglycemic therapy & $35(1.5)$ \\
Cyproheptadine & $30(1.2)$ \\
Octreotide & $29(1.2)$ \\
Lipid resuscitation therapy & $27(1.1)$ \\
Carnitine & $25(1.1)$ \\
Vitamin K & $20(0.8)$ \\
Methylene blue & $19(0.8)$ \\
Fab for digoxin & $19(0.8)$ \\
Pyridoxine & $16(0.7)$ \\
2-PAM & $6(0.2)$ \\
Hydroxocobalamin & $6(0.2)$ \\
Dantrolene & $4(0.2)$ \\
Ethanol & $3(0.1)$ \\
Bromocriptine & $3(0.1)$ \\
Nitrites & $3(0.1)$ \\
Anticoagulation reversal & $1(<0.1)$ \\
Andexanet & $1(<0.1)$ \\
Botulinum antitoxin & $1(<0.1)$ \\
Factor replacement & $1(<0.1)$ \\
Protamine & $1(<0.1)$ \\
Silimarin & $1(<0.1)$ \\
Total & $2410(100)$ \\
& \\
&
\end{tabular}

${ }^{\mathrm{a}}$ Percentages are based on the total number of antidotes administered $(N=2410) ; 1889$ (26.3\%) Registry cases received at least one antidote. Cases may have involved the use of multiple antidotes

\section{Non-pharmacologic Supportive Care}

Table 41 presents non-pharmacologic supportive care treatments reported to the Registry in 2019. The top two agents, IV fluid resuscitation (72.6\%) and intubation/ventilatory

Table 39 Antivenom therapy.

\begin{tabular}{ll}
\hline & $N(\%)^{\mathrm{a}}$ \\
\hline Crotalidae polyvalent immune fab (ovine) & $167(73.9)$ \\
Crotalidae immune fab & (equine) \\
Other snake antivenom & $45(19.9)$ \\
Spider antivenom & $9(4.0)$ \\
Scorpion antivenom & $3(1.3)$ \\
Total & $2(0.9)$ \\
\end{tabular}

${ }^{a}$ Percentages are based on the total number of antivenom treatments administered $(N=226) ; 196$ Registry cases $(2.7 \%)$ received at least one antivenom treatment. Cases may have involved the use of more than one antivenom 
Table 40 Supportive care- pharmacologic.

\begin{tabular}{ll}
\hline & $N(\%)^{\mathrm{a}}$ \\
\hline Benzodiazepines & $1615(51.2)$ \\
Opioids & $410(13.0)$ \\
Vasopressors & $316(10.0)$ \\
Antipsychotics & $225(7.1)$ \\
Glucose $>5 \%$ & $151(4.8)$ \\
Anticonvulsants & $99(3.1)$ \\
Neuromuscular blockers & $87(2.8)$ \\
Antihypertensives & $82(2.6)$ \\
Steroids & $63(2.0)$ \\
Beta-blockers & $40(1.3)$ \\
Albuterol and other bronchodilators & $39(1.2)$ \\
Antiarrhythmics & $24(0.8)$ \\
Vasodilators & $6(0.2)$ \\
Total & $3157(100)$
\end{tabular}

${ }^{a}$ Percentages are based on the total number of pharmacologic interventions $(N=3157) ; 2290$ Registry cases $(31.9 \%)$ received at least one pharmacologic intervention. Cases may have involved the use of multiple interventions

management (23.9\%), remain unchanged from last year and represent the large majority of agents in this category. ${ }^{3}$

\section{Chelation Therapy Administered}

Table 42 presents data on chelation therapy administered. There were 20 cases involving chelation reported in 2019, made up largely by DMSA (70.0\%) and deferoxamine $(20.0 \%)$.

Table 41 Supportive care — nonpharmacologic.

\begin{tabular}{ll}
\hline & $N(\%)^{\mathrm{a}}$ \\
\hline IV fluid resuscitation & $2560(72.6)$ \\
Intubation/ventilatory management & $843(23.9)$ \\
CPR & $43(1.2)$ \\
Transfusion & $30(0.9)$ \\
ECMO & $16(0.5)$ \\
Hyperbaric oxygen & $10(0.3)$ \\
Cardioversion & $8(0.2)$ \\
Pacemaker & $8(0.2)$ \\
Therapeutic hypothermia & $8(0.2)$ \\
Cardiopulmonary bypass & $1(<0.1)$ \\
Total & $3527(100)$ \\
\hline
\end{tabular}

$C P R$ cardiopulmonary resuscitation, ECMO extracorporeal membrane oxygenation

${ }^{\text {a }}$ Percentages are based on the total number of treatments administered $(N=3527) ; 2822$ Registry cases $(39.3 \%)$ received at least one form of nonpharmacologic treatment. Cases may have involved the use of multiple forms of nonpharmacologic treatment
Table 42 Chelation therapy.

\begin{tabular}{lc}
\hline & $N(\%)^{\mathrm{a}}$ \\
\hline DMSA & $14(70.0)$ \\
Deferoxamine & $4(20.0)$ \\
EDTA & $1(5.0)$ \\
Dimercaprol & $1(5.0)$ \\
Total & $20(100)$ \\
\hline
\end{tabular}

DMSA dimercaptosuccinic acid, EDTA ethylenediamine-tetraacetic acid

${ }^{\text {a }}$ Percentages are out of the total number of chelation treatments administered $(N=20) ; 19$ Registry cases $(0.3 \%)$ received at least one form of chelation treatment. Cases may have received multiple chelation treatments

\section{Decontamination Interventions Administered}

Table 43 describes the 235 decontamination interventions administered. Activated charcoal again represented the significant majority $(81.3 \%)$ in this class. ${ }^{3}$

\section{Enhanced Elimination Interventions Administered}

Table 44 presents the enhanced elimination interventions reported. Hemodialysis for toxin removal (32.1\%), followed by urinary alkalinization (27.0\%) and continuous renal replacement therapy (20.9\%), topped the reported interventions in this class.

\section{Discussion}

This report describes the 10th year of data collected for the Toxicology Investigators Consortium Registry. After several years of down-trending numbers reported to the Registry, in 2019 , reported registry cases have increased from the previous year. ${ }^{3}$ Notably, this increase in case numbers was not at the expense of quality, as the Registry has continued to increase quality control measures each year.

Table 43 Supportive care-decontamination.

\begin{tabular}{ll}
\hline & $N(\%)^{\mathrm{a}}$ \\
\hline Activated charcoal & $191(81.3)$ \\
Whole bowel irrigation & $22(9.4)$ \\
Irrigation & $11(4.7)$ \\
Gastric lavage & $11(4.7)$ \\
Total & $235(100)$ \\
\hline
\end{tabular}

${ }^{\text {a }}$ Percentage based on the total number of decontamination interventions $(N=235)$; 221 Registry cases $(3.1 \%)$ received at least one decontamination intervention. Cases may have involved the use of multiple interventions 
Table 44 Enhanced elimination.

\begin{tabular}{ll}
\hline & $N(\%)^{\mathrm{a}}$ \\
\hline Hemodialysis (toxin removal) & $63(32.1)$ \\
Urinary alkalinization & $53(27.0)$ \\
Continuous renal replacement therapy & $41(20.9)$ \\
Hemodialysis (other indication) & $30(15.3)$ \\
Multiple-dose activation charcoal & $7(3.6)$ \\
Exchange transfusion & $2(1.0)$ \\
Total & $196(100)$ \\
\hline
\end{tabular}

${ }^{\text {a }}$ Percentages are based on the total number of treatments administered $(N=196) ; 177$ Registry cases $(2.5 \%)$ received at least one form of enhanced elimination

Although the Registry is not strictly population based, it represents a wide geographic distribution of cases evaluated in person by medical toxicologists. These data can be used in conjunction with data from other registries including the National Poison Data System to provide a more detailed picture of poisoning trends, novel exposures, and their public health implications.

Trends in novel exposures were not described in this report but are being collected and analyzed to be reported separately. A summary of the 10-year Registry experience will also be reported separately.

Table 45 ToxIC EVALI demographics, use patterns, and outcomes.

\begin{tabular}{ll}
\hline & $N(\%)$ \\
\hline Age & $\begin{array}{l}\text { Range 13-55 years } \\
\text { Mean 22.7 years }\end{array}$ \\
Sex & $29(65.9)$ \\
Male & $15(34.1)$ \\
Female & $38(86.4)$ \\
No prior pulmonary or cardiac disease & \\
Products vaped & $23(52.3)$ \\
THC only & $2(4.5)$ \\
Nicotine only & $15(34.1)$ \\
Both THC and nicotine & $1(2.3)$ \\
Flavoring only & $3(6.8)$ \\
Unknown & \\
Treatment & \\
BIPAP & $9(20.5)$ \\
Intubation & $13(29.5)$ \\
Extracorporeal membrane oxygenation & $2(4.5)$ \\
Chest tubes & $3(6.8)$ \\
Steroids & $39(88.6)$ \\
Intensive care unit admission & $19(43.2)$ \\
Death & $1(2.3)$ \\
Total & $44(100)$ \\
\hline
\end{tabular}

Overall, this annual report finds trends in agent classes, agents, demographics, types of encounters, clinical signs and symptoms, and treatments to be largely unchanged from previous years. Notable findings or trends in the Registry are discussed below.

Addiction medicine consults have been recorded in the Registry for two years and are increasing in number. ${ }^{3}$ Further details of addiction medicine consults are described below.

The relative opioid cases continued to increase in 2019, making the opioid class the second most common agent class reported to the Registry for the first time in Registry history. In fact, the opioid class nearly eclipsed the non-opioid analgesics as the most common class in the Registry this year and may do so in 2020 based on recent trends. Overall, this is consistent with the larger trend of increasing opioid exposures reported to the Registry over time.

Fentanyl continues to represent a growing percentage of the opioid class reported to the Registry, rising to the second most common opioid this year. Although tramadol numbers fell, other oral opioids such as oxycodone and hydrocodone remained stable in 2019.

Again in 2019, there was an increase in psychoactive substances reported. Similar to last year, this increase was largely driven by an increase in non-synthetic cannabinoids including marijuana. Combined, various cannabinoid agents represented $63.0 \%$ of the psychoactive class, trending up from $55.0 \%$ in 2018. ${ }^{3}$ Nicotine exposures have also shown a consistent increase over the last two years, with a relative doubling in 2019 compared to $2018{ }^{3,4}$ This is likely multifactorial but may in part be reflective of national trends of increased use of vaping tobacco and cannabinoid products.

\section{Vaping Exposures}

In July 2019, an epidemic of vaping-related respiratory failure was reported in Illinois and Wisconsin. ${ }^{6}$ The illness termed "ecigarette, or vaping, product-use associated lung injury", or EVALI, has affected thousands of patients, including otherwise healthy adolescents and young adults. The leading etiology of EVALI is THC oil contamination with vitamin E acetate $^{7}$; however the exact pathophysiology is unknown. With input from the Federal Drug Administration and Centers for Disease Control (CDC), the Toxicology Investigators Consortium (ToxIC) committee created an EVALI surveillance form to gather more information on these cases. Within a month of the Center for Disease Control and Prevention's Health Advisory release, on October 10, 2019, the EVALI form was incorporated into REDCap. Medical toxicologists submitted suspected vaping cases they treated from July 2019 onward. Seven toxic sites entered data on 44 patients between October 10 and December 31, 2019. See Table 45 for case details. 
Table 46 Intentional pharmaceutical misuse/abuse to "get high" by age group and sex/ gender.
Table 47 Intentional nonpharmaceutical misuse/abuse to "get high" by age group and sex/ gender.

\begin{tabular}{llll}
\hline & Female N $(\%)^{\mathrm{a}}$ & Male N $(\%)^{\mathrm{a}}$ & Transgender $^{\mathrm{d}} \mathrm{N}(\%)^{\mathrm{a}}$ \\
\hline Age 7-12 & & & \\
Antidepressants & $6(5.4)$ & $0(0)$ & $0(0)$ \\
Herbals/dietary supplements/vitamins & $2(1.8)$ & $0(0)$ & $0(0)$ \\
Cough and cold medication & $1(0.9)$ & $0(0)$ & $0(0)$ \\
Total & $9(8.0)$ & $0(0)$ & $0(0)$ \\
Age 13-18 & $5(4.5)$ & $4(3.6)$ & $0(0)$ \\
Antipsychotics & $2(1.8)$ & $2(1.8)$ & $\mathrm{M} \rightarrow \mathrm{F}: 1(0.9)$ \\
Cough and cold medication & $4(3.6)$ & $1(0.9)$ & $0(0)$ \\
Lithium & $6(5.4)$ & $1(0.9)$ & $\mathrm{F} \rightarrow \mathrm{M}: 1(0.9)$ \\
Miscellaneous & $17(15.2)$ & $8(7.1)$ & $\mathrm{F} \rightarrow \mathrm{M}: 1(0.9) ; \mathrm{M} \rightarrow \mathrm{F}: 1(0.9)$ \\
Total & & & \\
Age $\geq 19$ & $13(11.6)$ & $11(9.8)$ & $\mathrm{M} \rightarrow \mathrm{F}: 1(0.9)$ \\
Antidepressants & $9(8.0)$ & $5(4.5)$ & $0(0)$ \\
Cardiovascular & $7(6.3)$ & $5(4.5)$ & $0(0)$ \\
Diabetic medication & $16(14.3)$ & $9(8.0)$ & $0(0)$ \\
Miscellaneous & & $30(26.8)$ & $\mathrm{M} \rightarrow \mathrm{F}: 1(0.9)$ \\
Total & $45(40.2)$ & $38(33.9)$ & $3(2.7)$ \\
Misuse/abuse to "get high" total & $71(63.4)$ &
\end{tabular}

${ }^{\text {a }}$ Percent of total number of cases $(N=112)$ with misuse/abuse to "get high"

${ }^{\mathrm{b}}$ Includes diabetic medication, herbals/dietary supplements/vitamins, caustic, and cardiovascular

${ }^{\mathrm{c}}$ Includes antipsychotics, chemotherapeutic, household product, caustic, herbals/dietary supplements/vitamins, cough \& cold medication, antimicrobial, and gastrointestinal

${ }^{\mathrm{d}} \mathrm{M} \rightarrow \mathrm{F}$ is transgender male to female; $\mathrm{F} \rightarrow \mathrm{M}$ is transgender female to male

\begin{tabular}{llll}
\hline & Female $N(\%)^{\mathrm{a}}$ & Male $N(\%)^{\mathrm{a}}$ & Transgender $^{\mathrm{d}} N(\%)^{\mathrm{a}}$ \\
\hline Age 7-12 & & \\
Opioid & $0(0)$ & $1(0.2)$ & $0(0)$ \\
Total & $0(0)$ & $1(0.2)$ & $0(0)$ \\
Age 13-18 & $10(2.3)$ & $32(7.2)$ & \\
Psychoactive & $3(0.7)$ & $10(2.3)$ & $0(0)$ \\
Alcohol ethanol & $3(0.7)$ & $5(1.1)$ & $0(0)$ \\
Sympathomimetic & $2(0.5)$ & $13(2.9)$ & $0(0)$ \\
Miscellaneous & $18(4.0)$ & $60(13.5)$ & $0(0)$ \\
Total & & & $\mathrm{M} \rightarrow \mathrm{F}: 1(0.2)$ \\
Age $\geq 19$ & $46(10.4)$ & $87(19.6)$ & $0(0)$ \\
Opioids & $19(4.3)$ & $67(15.1)$ & $0(0)$ \\
Sympathomimetic & $17(3.8)$ & $50(11.3)$ & $0(0)$ \\
Psychoactive & $21(4.7)$ & $56(12.6)$ & $\mathrm{M} \rightarrow \mathrm{F}: 1(0.2)$ \\
Miscellaneous & & $260(58.7)$ & $\mathrm{M} \rightarrow \mathrm{F}: 1(0.2)$ \\
Total & $103(23.3)$ & $321(72.5)$ & \\
Total & $121(27.3)$ & &
\end{tabular}

Cases with age unknown were excluded from this table

${ }^{\text {a }}$ Percent based on total number of cases $(N=443)$ with misuse/abuse to "get high"

${ }^{\mathrm{b}}$ Includes opioids, toxic alcohol, amphetamine-like hallucinogen, plant and fungi, sedative hypnotic, and unknown

${ }^{\mathrm{c}}$ Includes ethanol, sedative hypnotic, amphetamine-like hallucinogen, toxic alcohol, analgesic, anesthetics, cough and cold, gases/vapors/irritants/dusts, hydrocarbons, diabetic, plants and fungi, unknown, not reported, and other ${ }^{\mathrm{d}} \mathrm{M} \rightarrow \mathrm{F}$ is transgender male to female; $\mathrm{F} \rightarrow \mathrm{M}$ is transgender female to male 


\section{Self-Harm Intent}

Cases in which there was intent for self-harm are described in detail in this report (Tables 7, 8, 10, and 11). Self-harm cases represented $36.6 \%$ of Registry cases and included polysubstance ingestions in nearly half of reported cases (44.3\%). The analgesic class was the most common agent class reported both for cases of self-harm and to the Registry as a whole. In general, the top agent classes reported with intent for self-harm followed closely with that of the general Registry with one major exception: opioids. The opioid class represented $13.4 \%$ of Registry agents (second most common class) but only $3.2 \%$ of agents in the category of self-harm intent (ninth most common class). This highlights the likely accidental nature of many serious opioid overdoses. Focusing opioid education and intervention efforts on preventing accidental overdoses continues to warrant substantial attention.

Although women represented half of Registry cases, they were over-represented (65.0\%) in cases of self-harm compared to men. Also interesting was the significant proportion of cases reporting self-harm in teens 13-18 years of age $(39.5 \%)$, nearly twice the representation of this age group compared to the general Registry (20.6\%). In teens, analgesics, antidepressants, and anticholinergics represented the majority of agents involved in self-harm (61.8\%). Opioids, however, represented a distinctly low percentage of agents in this group (1.2\%). There was a marked age-related difference in self-harm attempts with opioid and benzodiazepine exposures, both of which increased with age. For opioids, there were very few self-harm cases in children $(0.7 \%)$ and teens $(1.2 \%)$, but a moderate number in adults $(4.0 \%)$ and significant number in older adults (13.3\%). For benzodiazepines, there were moderate numbers in children $(5.3 \%)$ and teens $(4.4 \%)$, but more significant numbers in adults $(12.8 \%)$ and older adults $(14.5 \%)$. The reason for this is uncertain; however, access to medications may play a role. Children and teens are less commonly prescribed opioids or benzodiazepines but may have easier access to over-the-counter medications like analgesics and anticholinergics, and some teens may be prescribed antidepressants. Parents' awareness of dangers of opioids and benzodiazepines may be higher than for other prescription medications and may influence safe storage and supervision practices. As such, increased safety measures may be needed, such as keeping these medications out of reach of children and teens.

Interestingly, the percent of deaths in cases of intent for self-harm $(1.1 \%)$ did not differ significantly from the Registry as a whole (1.3\%).

\section{Substance Use Disorder and Gender}

Encounters for intentional misuse/abuse of a substance without intent at self-harm, and with "attempt to illicit a pleasurable sensation (e.g. to get 'high')" and/or with "attempt to avoid withdrawal" are reported by age group and sex/ gender in Table 46 (pharmaceuticals) and Table 47 (nonpharmaceuticals).

Females represented $63.4 \%$ of cases where pharmaceuticals were used to "get high" and $80 \%$ of cases where pharmaceuticals were used to avoid withdrawal. Among cases where non-pharmaceuticals were misused/abused: males represented $72.3 \%$ of cases where intent was to "get high," $78.9 \%$ of cases where intent was to avoid withdrawal, and $63.8 \%$ of cases where use was both to "get high" and avoid withdrawal. Some substances reported did not necessarily have a mechanism of action consistent with the intent of the exposure which may indicate a lack of understanding of potential effects.

Drug concealment cases were $83.9 \%$ male. Males also comprised the majority of cases of ethanol abuse (64.1\%); there were no ethanol abuse cases reported in transgender individuals. $82.6 \%$ of ethanol abuse cases were in the age group 19-65 years.

Males comprised the majority of cases of ethanol (77\%) and cocaine/amphetamine (66.7\%) withdrawal cases. Opioid and sedative-hypnotic withdrawal cases did not demonstrate a gender predominance $50.7 \%$ and $51.5 \%$ female, respectively). There were no cases of withdrawal in transgender individuals.

\section{Addiction Medicine and Substance Use Disorder Consultation}

This report marks the second year that the ToxIC Registry collected data to specifically evaluate the care of patients with addiction (ADM) and substance use disorder (SUD). Notably, these data identify cases in which the primary reason for consultation was related to addiction and not another toxicologic indication. In 2019, there were 476 cases in which the primary reason for consultation was addiction, nearly a doubling from the 244 cases in 2018. The majority of these cases ( 323 cases, $69 \%$ ) arose as consults from inpatient or admitting services, followed by consults from the Emergency Department (142 cases, $30 \%$ ). The mean age was 42 years and $44 \%$ were female. Opioid agonist therapy initiation was the most common reason for consultation (62\%) followed by pain management (83 cases, 18\%), counseling (44 cases, 9\%), alcohol dependence pharmacotherapy (31 cases, $7 \%$ ), and opioid antagonist therapy initiation ( 6 cases, $1 \%)$. This breakdown is similar to that of 2018. Incomplete data resulted in some categories not adding to $100 \%$.

In addition to ADM and SUD cases, there were an additional 92 cases primarily related to alcohol abuse and 407 to withdrawal. Of the withdrawal cases, $183(45 \%)$ involved ethanol, 152 (37\%) opiates, 48 (12\%) sedative-hypnotics, 18 (4\%) other, and $6(1 \%)$ stimulants. This is similar to 2018 in which the majority of withdrawal cases were secondary to 
ethanol and opiate use. Overall, these numbers represent a dramatic increase in bedside medical toxicology expertise consultation for substance use disorder, pain management, and addiction.

\section{Limitations}

The ToxIC Registry is a unique prospective database of cases in which bedside consultation is performed by medical toxicologists, allowing an informed relationship between exposures and clinical outcomes. There are, however, some limitations within the Registry. One of these is a possible bias towards inclusion of more severe case presentations, since cases are only included if they undergo subspecialty bedside consultation. Cases for which a medical toxicology consultation was not requested are not reported and may represent a group with less severe illness. Therefore, the Registry likely represents a different population from other data sources such as poison control centers. There may also be a disproportionate number of certain cases reported based on regional variations in drug use, abuse, and other toxic exposures. The ToxIC Registry includes sites from multiple, diverse locations, but the entire country is not uniformly represented. Larger academic medical centers with greater amounts of medical toxicology faculty may be over-represented in the database.

Additionally, there may be a reporting bias towards more complicated or interesting cases at the level of individual sites. Although the specific intent of the Registry, as defined in written agreements with all sites, is to obtain a consecutive sample of all cases at a given site, individual cases may be missed. Data regarding substances of exposure or species of envenomation relies heavily on patient self-report and may be misclassified. Willingness to disclose this information may be particularly true of illicit drug exposure. Lastly, the Registry strives to continually improve the quality of data collected. While member sites are instructed to complete all applicable data fields, there are still a number of cases and data fields with incomplete information. This remains an issue for collection of race and ethnicity, for example. Efforts continue to support quality data collection and follow up on missing data where applicable.

\section{Conclusions}

The ToxIC Registry continues to be unique among databases in that it represents prospective data collected from cases evaluated at the bedside by medical toxicologists. Although this feature limits extrapolation to the population as a whole, it increases the potential for high quality data and for increased correlation between exposure cases and clinical findings. Continued quality improvement and surveillance efforts remain areas of focus for the Registry.

Acknowledgments Toxicology Investigators Consortium (ToxIC) Study Group Collaborators:

Akpunonu PA, Amaducci A, Barbuto AF, Baum RA, Baumgartner K, Beuhler MC, Billington M, Boyle KL, Burns MM, Carey JL, Carpenter J, Ceretto V, Chary MC, Chenoweth JA, Colby DK, De Olano J, Devgun J, Eisenga BH, Fishburn S, Ford JB, Froberg B, Ganetsky M, Gorodetsky R, Greene SC, Griswold M, Hendrickson RG, Hughes AR, Jacob J, Judge BS, Kao L, Koons AL, Leikin JB, Lo CY, Lopez AM, McFalls J, McKay C, Meaden CW, Nacca N, Nanagas K, Niruntarai S, Obilom C, O'Connor AD, Othong R, Reibling ET, Riley BD, Santos C, Schult R, Seifert SA, Shao S, Sidlak A, Smolinske SC, Steck A, Surmaitis RM, Thompson M, Warrick BJ, Wolk BJ.

We also wish to thank study coordinators: Beuhler PM, Falter T, Ford J, Garcia DA, Hart K, Iocco MG, Katz KD, Licata JB, Menegazzi R, Padilla-Jones A, Phan T, Ramirez A, Roosta M, and Vandenberg M.

Sources of Funding The Toxicology Investigators Consortium received funding from the US National Institute of Drug Abuse 1RO1DA03731702 and data-sharing contracts with the U.S. Food and Drug Administration and BTG International, Inc. (North America).

Previous Presentation of Data These data have not been previously presented.

\section{Compliance with Ethical Standards}

Conflict of Interest None

\section{References}

1. Brent J, Wax PM, Schwartz T, et al. The toxicology investigators consortium case registry-the 2010 experience. J Med Toxicol. 2011;7(4):266-76.

2. Farrugia LA, Rhyee SH, Calello DP, et al. The toxicology investigators consortium case registry-the 2016 experience. J Med Toxicol. 2017;13(3):203-26.

3. Spyres MB, Farrugia LA, Kang MA, et al. The toxicology investigators consortium case registry-the 2018 experience. J Med Toxicol. 2019;15(4):228-54.

4. Farrugia LA, Rhyee SH, Campleman SL, et al. The toxicology investigators consortium case registry-the 2017 experience. J Med Toxicol. 2018;14(3):182-211.

5. Farrugia LA, Rhyee SH, Campleman SL, et al. The toxicology investigators consortium case registry-the 2015 experience. J Med Toxicol. 2016;12(3):224-47.

6. Layden JE, Ghinai I, Pray I, et al. Pulmonary illness related to Ecigarette use in Illinois and Wisconsin - preliminary report. N Engl J Med. 2019.

7. Cao DJ, Aldy K, Hsu S, McGetrick M, Verbeck G, De Silva I, et al. Review of health consequences of electronic cigarettes and the outbreak of electronic cigarette, and vaping, product use-associated lung injury. J Med Toxicol. 2020;16(3):295-310.

Publisher's Note Springer Nature remains neutral with regard to jurisdictional claims in published maps and institutional affiliations. 


\section{Affiliations}

Meghan B. Spyres ${ }^{1,2} \cdot$ Lynn A. Farrugia $^{3} \cdot$ A. Min Kang ${ }^{2,4} \cdot$ Kim Aldy $^{5,6} \cdot$ Diane P. Calello ${ }^{7} \cdot$ Sharan L. Campleman ${ }^{6}$. Shao $\mathrm{Li}^{6} \cdot$ Gillian A Beauchamp ${ }^{8}$. Timothy Wiegand ${ }^{9} \cdot$ Paul M. Wax ${ }^{5,6} \cdot$ Jeffery Brent ${ }^{10} \cdot$ On behalf of the Toxicology Investigators Consortium Study Group

1 Department of Emergency Medicine, University of Arizona College of Medicine, Phoenix, AZ, USA

2 Department of Medical Toxicology, Banner - University Medical Center, 1012 E Willetta Street, Fl 2, Phoenix, AZ 85006, USA

3 Hartford Hospital and University of Connecticut School of Medicine, 80 Seymour Street, Hartford, CT 06102, USA

4 Departments of Medicine and Child Health, University of Arizona College of Medicine, Phoenix, AZ, USA

5 University of Texas Southwestern Medical School, 5323 Harry Hines Boulevard, Dallas, TX 75390, USA

6 American College of Medical Toxicology, 10645 N Tatum Blvd., Suite 200-111, Phoenix, AZ 85028, USA
7 New Jersey Medical School, Rutgers, The State University of New Jersey, 140 Bergen Street, Suite G1600, Newark, NJ 07101-1709, USA

8 Lehigh Valley Health Network Department of Emergency Medicine and Hospital Medicine, Division of Medical Toxicology, USF Morsani College of Medicine, Lehigh Valley Campus, Cedar Crest Boulevard \& I-78, Allentown, PA 18101, USA

9 University of Rochester Medical Center and Strong Memorial Hospital, 601 Elmwood Ave, Rochester, NY 14642, USA

10 University of Colorado School of Medicine, 13001 E 17th Pl, Aurora, CO 80045, USA 